\title{
Metallicities and activities of southern stars ${ }^{\star}, \star \star$
}

\author{
J. S. Jenkins ${ }^{1,2}$, H. R. A. Jones ${ }^{2}$, Y. Pavlenko ${ }^{2}$, D. J. Pinfield ${ }^{2}$, J. R. Barnes ${ }^{2}$, and Y. Lyubchik ${ }^{2}$ \\ 1 Department of Astronomy and Astrophysics, Pennsylvania State University, University Park, PA 16802, USA \\ e-mail: jjenkins@astro.psu.edu \\ 2 Centre for Astrophysics, University of Hertfordshire, College Lane Campus, Hatfield, Hertfordshire, AL10 9AB, UK
}

Received 4 September 2007 / Accepted 4 April 2008

\section{ABSTRACT}

\begin{abstract}
Aims. We present the results from high-resolution spectroscopic measurements to determine metallicities and activities of bright stars in the southern hemisphere.

Methods. We measured the iron abundances ([Fe/H]'s) and chromospheric emission indices $\left(\log h R_{H K}^{\prime}\right)$ of 353 solar-type stars with $V=7.5-9.5$. $[\mathrm{Fe} / \mathrm{H}]$ abundances are determined using a custom $\chi^{2}$ fitting procedure within a large grid of Kurucz model atmospheres. The chromospheric activities were determined by measuring the amount of emission in the cores of the strong CaII HK lines.

Results. Our comparison of the metallicity sample to other $[\mathrm{Fe} / \mathrm{H}]$ determinations was found to agree at the \pm 0.05 dex level for spectroscopic values and at the \pm 0.1 dex level for photometric values. The distribution of chromospheric activities is described by a bimodal distribution, agreeing with the conclusions from other works. Also an analysis of Maunder minimum status was attempted, and it was found that $6 \pm 4$ stars in the sample could be in a Maunder minimum phase of their evolution and hence the Sun should only spend a few per cent of its main sequence lifetime in Maunder minimum.
\end{abstract}

Key words. stars: abundances - stars: activity - stars: atmospheres - stars: planetary systems

\section{Introduction}

High-resolution spectroscopy is an important tool for understanding stars in the local neighbourhood and can give an insight into various fundamental properties of the stars themselves (e.g. Edvardsson et al. 1993; Wright 2004a; Valenti \& Fischer 2005). Indeed, knowledge of stellar fundamental properties can also have a direct bearing on other facets of stellar astrophysics such as the potential for discovering planetary systems. After only three exoplanets had been discovered, Gonzalez (1997) noticed all were orbiting stars with a higher metal content than the Sun. This trend has continued and been confirmed by various authors, most recently by Fischer \& Valenti (2005) who have shown that the probability of discovering an exoplanet around a solar-type star is proportional to the stellar metal abundance following a power law described by $0.03 \times 10^{2.0[\mathrm{Fe} / \mathrm{H}]}$, consistent with the predictions made by the core accretion models of planet formation. Along with a star's metallicity, another important feature is the star's chromospheric activity.

Stellar chromospheric activity is intimately related to the stellar dynamo, magnetism and the stellar rotation (Middelkoop 1982a; Middelkoop 1982b; Rutten 1984). Since the measurement of activity gives us a feel for the motions on both large and small scales in a star's atmosphere, it can be used to select the most inactive and hence stable stars to scrutinize for exoplanets. It has been found that chromospheric activity can mask, or in some cases exhibit planetary radial-velocity signatures (e.g. Queloz et al. 2001; Henry et al. 2002). Therefore it seems

* Based on observations made with the ESO telescopes at the La Silla Paranal observatory under programme ID's 076.C-0578(B) and 077.C-0192(A).

$\star \star$ Table 4 is only available in electronic form at the CDS via anonymous ftp to cdsarc.u-strasbg.fr (130.79.128.5) or via http://cdsweb.u-strasbg.fr/cgi-bin/qcat?]/A+A/485/571 prudent for any radial-velocity planet search programme aiming to reach precisions of a few $\mathrm{m} \mathrm{s}^{-1}$ to select the least active stars. We began a radial-velocity project to detect both hot Jupiter-type exoplanets and lower-mass hot Saturns and Neptunes that have the potential to transit their host star. In Sect. 2 we discuss all observations and reduction methods. In Sect. 3 the methodology for extracting the spectroscopic information is explained: both the activity analysis and the stellar photospheric properties, such as metallicity abundance. Section 4 compares the values determined here with those of other works in the literature. In Sect. 5 the results are discussed in terms of both the current status of exoplanets and stellar evolution. Lastly, in Sect. 6 we discuss the conclusions of the project and plans for future work using this dataset.

\section{Observations and reduction}

All target stars and calibration data were observed using the Fibre-fed Extended Range Optical Spectrograph (FEROS) mounted on the MPG/ESO - 2.2 m telescope on the La Silla site in Chile. To gain access to the entire southern sky the run scheduling was split across two independent observing runs seven months apart. The runs were three nights in length, from 02 to 05 of February and September 2006. This culminated in over 350 stellar observations with exposure times in the range $120-480 \mathrm{~s}$. These exposure times gave rise to $\mathrm{S} / \mathrm{N}$ ratios of 100-200 in the continuum around the iron line at $7500 \AA$ and a median of $\sim 60$ at the CaHK lines (3955 $\AA$ ), with only a small tail of low $\mathrm{S} / \mathrm{N}$ stars reaching down to values of 30 . All calibration files needed for reducing the stellar spectra (flat-fields, bias and arc frames) were obtained at the beginning and end of each night's observing, following the standard ESO calibration plan.

The reduction of all spectra followed the standard reduction techniques. First, the bias signal was removed from each 
individual frame. The overscan region was subtracted out and trimmed off the image so as not to confuse any of the extraction algorithms. FEROS echelle data shows strong curvature in the 2D echelle image and, due to the use of an image slicer, has a complex slit cross-profile. The curved echelle profile was straightened by fitting polynomials along the orders. These were then precisely traced using a well exposed Halogen flatfield image to follow the lamp light path. Due to the half moon-like pattern of the sliced beams the orders were smoothed in the spatial direction to create a single smooth order. This allowed the tracing algorithm to properly follow the centre of the order and not vary between the two sliced peaks. The traces were then clipped to remove any stray pixel counts and to help tighten up the trace and then object and background apertures were selected. To correct for the CCD pixel-to-pixel response we obtained 10+ flatfields with $\mathrm{S} / \mathrm{N}$ ratios close to 1000 each morning and evening. Each pixel was median combined together to create a master flatfield frame. The master flats were then used to calculate the balance factors by smoothing in the dispersion direction and dividing the smoothed flat by the master flat. These balance factors were then multiplied into each science frame to flat field each image. ThAr+Ne and ThArNe lamps were used to wavelength calibrate all the data. The sky background was negligible; however the scattered light was still removed. This was done by setting the dekker limits large enough to include wide interorder spacing regions and then a low-order polynomial was used to sample any gradients along the orders. A second low-order polynomial was then used to fit to the total intensity over the whole order to better model the scattered light. The profile of each order was measured by sub-sampling each order profile individually using Gaussians. This model was used to extract the object with an optimal extraction algorithm. The data were then binned to a linear wavelength step of $0.03 \AA /$ pixel, or a mean velocity resolution of $1.42 \mathrm{~km} \mathrm{~s}^{-1} /$ pixel, and shifted into the rest frame by cross-correlating with an observation of HD 102117 (G6V), which has been shown to exhibit a radial-velocity variation $<20 \mathrm{~ms}^{-1}$ (Jones et al. 2002).

\section{Methodology}

\subsection{Activities}

The methodology used to extract these high-resolution activity indices is similar to that employed in previous works by the Anglo-Australian Planet Search e.g. Tinney et al. (2002), Jenkins et al. (2006, hereafter J06) and Mount Wilson (Duncan et al. 1991) projects. Four passbands situated in a well concentrated region of the UV were used for flux measurements. Figure 1 shows the part of the spectrum used for all stars. This particular spectrum is of HD 59100, which is similar to the Sun with a spectral type of $\mathrm{G} 2 \mathrm{~V}$ and has a final activity of $\log R_{H K}^{\prime}=$ -4.92 . All the four passbands used to make the activity measurements have been shown for reference. Each of the individual lines apparent in Fig. 1 are relatively weaker spectral features, not noise.

\subsection{1. $S_{M W}$ calibration}

Tinney et al. (2002), J06 and Wright (2004a) have shown that linear calibrations are needed to calibrate high-resolution activity indices onto the MW system of measurements (Duncan et al. 1991). The setup employs four separate passbands centered on and around the CaII HK lines. The passbands used to estimate the mean flux in the continuum $(V \& R)$ are both square and

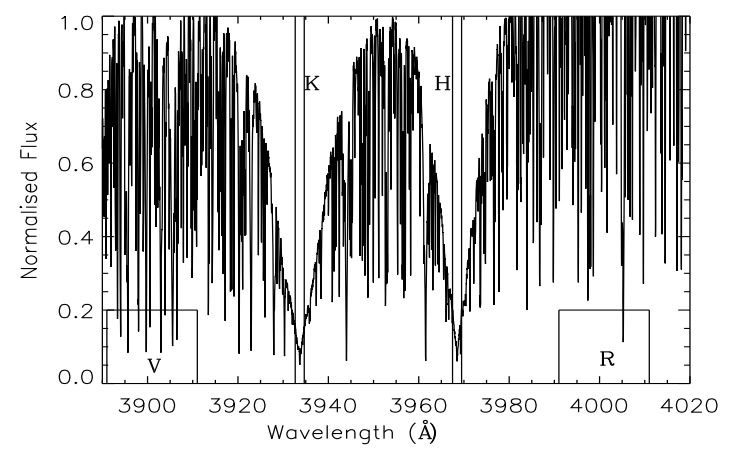

Fig. 1. The extracted FEROS spectra of the star HD 59100 around the CaII $\mathrm{K}$ and $\mathrm{H}$ lines. The star has a spectral type of G2V and is chromospherically quiet $\left(\log R_{H K}^{\prime}=-4.92\right)$. All the four passband regions $(V$, $K, H$ and $R$ ) have been highlighted on the plot. The spectrum has been normalised to the continuum region 3991-4011 A which represents the $\mathrm{R}$ passband. All other features are relatively much weaker absorption lines, not noise.

centered at wavelengths of $3891 \AA$ and $4001 \AA$ respectively. Whereas the passbands used to obtain the mean flux in the $\mathrm{K}$ and $\mathrm{H}$ line cores have triangular profiles with full-width half maximum's $(F M H M)$ of $1.09 \AA$ and are centered on the lines themselves at wavelengths of $3933.664 \AA$ and $3968.470 \AA$ respectively.

The FEROS activity indicator, $S_{\text {FEROS }}$, is determined by

$S_{\mathrm{FEROS}}=\frac{N_{H}+N_{K}}{N_{R}+N_{V}}$

Here in Eq. (1), the $N_{i}$ is the number of counts in each bandpass (where $i=H, K, V$ and $R$ ) and hence this provides a measure of the ratio of the flux in the CaII line cores to that of the continuum. When this index was created for use at Mt. Wilson it was tailored towards use with the photometer and passband wheel there and also employed a normalising factor, however by use of a similar, yet artificial setup, this procedure works very well for extracting activity indices of bright solar-type stars.

A number of stars from Duncan et al. (1991) were selected for use as calibrators onto the MW system, these are shown in Table 3. The table shows each of the calibrating star's Johnson $V$ magnitude and $B-V$ colour from Hipparcos, along with the $S$ index before $\left(S_{\text {FEROS,a }}\right)$ and after $\left(S_{\text {FEROS,b }}\right)$ calibration on the MW system, the FEROS and MW $\log R_{H K}^{\prime}$ values and the final metallicities. For any activity study, the selected comparison stars should not introduce any offset into the final $S$-indices, however this remains difficult due to the lack of long-term monitoring data in the literature for stars in the southern hemisphere. Two of our calibration stars, HD 1835 and HD 10700, have recently had their long-term variability and activity trends published. Lockwood et al. (2007) have shown that HD 1835 did exhibit variation at the level of $\sim \pm 0.05$ between 1985 and 2002. However, the star only spent a limited time at these extremes and was mostly found to be only $\sim \pm 0.02-0.03$ from the mean activity value. Hall et al. (2007) found a mean $S$-index for this star of 0.362 using 82 observations over a period of $\sim 11$ years, and this mean is 0.012 larger than the final computed value here. HD 10700 was also measured by Hall et al. and they found the mean $S$-index to be 0.175 over 10 years and 67 observations, which is close to the value of 0.179 found here (i.e. two measurements of 0.178 and 0.180 ) within the uncertainties. Figure 2 shows the linear fit between the FEROS and the published MW values. The fit has a gradient of $1.144 \pm 0.001$ and an intercept of $0.018 \pm 0.006$. The overall scatter of the points 


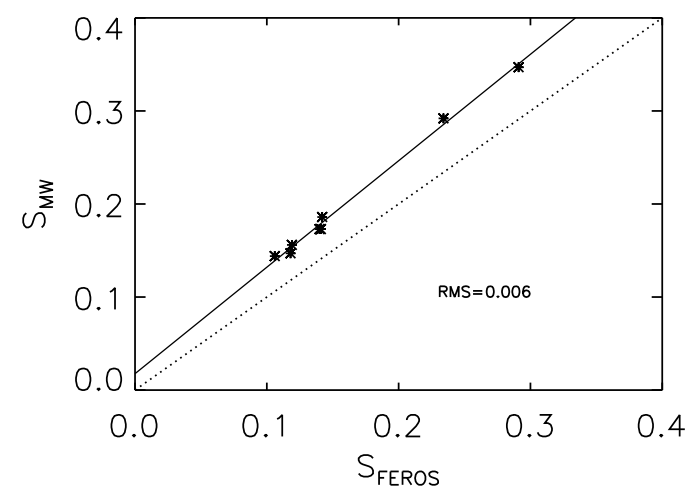

Fig. 2. This plot shows the linear fit applied to the FEROS spectra to convert the dataset to the MW system of measurements. All stars used as calibrators were taken from the MW project. The fit shows an increasing linear trend with increasing $S$-index. The overall scatter of the points is highlighted on the plot, and with a value of 0.006 , this is in agreement with the findings of Tinney et al. (2002), Wright (2004a) and J06 who all calibrated using high-resolution data and setups similar to the MW project.

around the linear trend is 0.006 , which is similar to the scatter found by J06. This low level of scatter gives confidence in the robust nature of the reduction and analysis procedure. The calibration error is found to be consistent with the removal of any of the data points.

Wright (2004a) and J06 used measurements of the stable star $\tau$ Ceti (HD 10700) as a proxy for the random uncertainty introduced in the reduction procedure. As $\tau$ Ceti has been shown to be extremely stable (Baliunas et al. 1995) this is a useful tool for estimating the uncertainty introduced in the reduction procedure. Only two measurements of this star were possible due to the star's position and bad weather, and hence it could only be used as a weak uncertainty estimator. The measurements were obtained over two separate nights and have shown the stability of the reduction. The computed $S$-index for both measurements are 0.178 and 0.180 , indicating that the procedure is robust. As the reduction uncertainty is weakly estimated here, the rms scatter can be used as a first approximation of the total uncertainty, and also by comparison with the findings from Wright (2004a) and J06 where both find uncertainties of $\sim 3-5 \%$. We estimate that the total error included in each individual $S$ activity indicator is around $3-5 \%$.

\subsection{2. $\log R_{H K}^{\prime}$ fit}

The measured $S$-index contains photospheric information, whereas for activity analysis one wants exclusively chromospheric information, therefore the methodology of Noyes et al. (1984a) has been used to remove this photospheric component. This method normalizes the flux to the bolometric luminosity of the star by use of empirical relations (see Noyes et al., for equations). Figure 3 shows the final $\log R_{H K \text {,FEROS }}^{\prime}$ values for all stars used to calibrate onto the MW system of measurements. The plot highlights the close relationship between the published MW values from Duncan et al. (1991) and these values. A linear best-fit trend is applied to the data and this is shown by the solid line in the plot. The gradient of this trend is $0.981 \pm$ 0.003 and has a negative intercept of $-0.092 \pm 0.250$. The slope of this fit is in agreement with that found by J06. No other calibration is necessary. The rms scatter here is 0.034 , compared to $\sim 0.04$ at the AAT. Due to the number of uncertainties

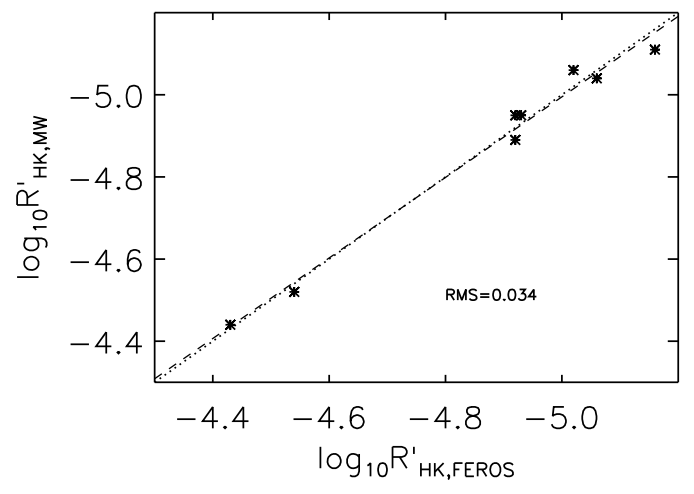

Fig. 3. The final $\log R_{H K}^{\prime}$ values for the FEROS calibration dataset against the values published in Duncan et al. (1991) (MW). There appears a tight fit around the 1:1 relation, which is shown by the dotted line. The dashed line represents the best-fit linear trend to this data and the tight relationship means no further calibration is required. The rms scatter around the fit is shown in the plot and is found to be 0.034 .

that can be introduced in the reduction procedure, such as the scattered light removal, blaze extraction, flatfielding etc., we believe that this rms is the best overall estimate of the uncertainties associated with each individual activity measurement. When we compare the final Hall et al. $\log R_{H K}^{\prime}$ values for the two calibration stars HD 1835 and HD 10700 against the values presented here, we see good agreement between the two within the uncertainties expressed i.e. $-4.41 /-4.43$ and $-4.94 /-4.91$ respectively. All final $\log R_{H K \text {,FEROS }}^{\prime}$ indices, along with their associated $S$-indices, Johnson colours and visual magnitudes from Hipparcos (Perryman et al. 1997) are shown in Table 4.

\subsection{Metallicities}

When attempting to obtain accurate metallicity abundances $([\mathrm{M} / \mathrm{H}])$ of solar-type stars some prior knowledge of the stellar envelope conditions can be useful. Various important stellar parameters are input into spectral synthesis codes to model stellar spectra, these are the effective temperature $\left(T_{\mathrm{EFF}}\right)$, the stellar surface gravity $(\log g)$, the microturbulence parameter $\left(v_{\text {mic }}\right)$, the macroturbulent velocity $\left(v_{\text {mac }}\right)$ and the stellar rotational velocity $\left(v_{\text {rot }}\right)$. All of these parameters serve to either alter the line profiles and/or strengths of elemental abundances in the stellar envelope and can have marked effects on any abundance determination.

\section{3. $T_{E F F}$ determination}

Various tables consisting of numerous stellar parameters and calibrations have been published in the literature. For example, Strömgren $u b v y$-photometry for a host of stars can be found in Hauck \& Mermilliod (1998) and using calibrations from Olsen (1984) the effective temperatures and surface gravities can be found. Also the catalogue of Nordström et al. (2004) has an extensive list of effective temperatures for over 14000 stars. Nordström et al. (2004) has also published the Strömgren derived $[\mathrm{Fe} / \mathrm{H}]$ values of all stars in their catalogue and, along with the calibrations of Haywood (2002) for solar-type stars in the Hauck \& Mermilliod (1998) catalogue, a large library of Strömgren $[\mathrm{Fe} / \mathrm{H}]$ can be compiled. A large number of stars in this study have measured Strömgren indices. Such measurements only allow for an estimate of the overall metallicity abundance. Spectral analysis is a more accurate technique for the determination of the overall metallicity abundance, albeit at 
greater labour, as the information contained in each line is measured individually and then combined. Whereas using photometry means losing all information on each individual element and relying on the stability and accuracy of the photometry and also the calibration of the scale with stellar parameters. Hence, all stars with Strömgren indices were kept in this observing programme but priority was given to stars with no information at all. Also as most stars in this project did not have measured Strömgren indices a different method was needed to obtain the stellar temperatures.

Recently a common method of temperature determination is by use of fitting the stellar spectra themselves (e.g., see Santos et al. 2003; Santos et al. 2004; Valenti \& Fischer 2005). However this method can be quite computer intensive and quantifying the errors can be quite difficult. A simpler method of obtaining the effective temperature is by using photometric colour-effective temperature relations (e.g., Smith 1995, Eq. (8.9)), which was recently employed by Bond et al. (2006) to determine the effective temperatures of stars on a full target list. The Infrared Flux Method (IRFM) (Blackwell \& Lynas-Gray 1994) was the calibration chosen to generate the effective temperatures using the Hipparcos $V$ magnitude and the 2MASS $K_{\mathrm{s}}$ magnitude of all these stars. Since the 2MASS $K_{\mathrm{s}}$ is a shorter band measurement than the typical Johnson $K$-band, a magnitude correction taken from Carpenter (2001) was used to correct the $K_{\text {s }}$ magnitude for application to the IRFM. The calibration from Blackwell \& Lynas-Gray was used to generate the final effective temperatures of all stars. The $V-K$ colours have been found to be more reliable as a temperature indicator due to less metallicity independence, reduced line blanketing, the colour variation is less susceptible to surface gravity (Alonso et al. 1996) and the colours span a wider range of values compared to $B-V$ colours, giving a tighter sequence.

\subsubsection{Surface gravity}

The surface gravity $(\log g)$ of stellar objects is another parameter that can alter stellar line strengths, therefore altering the final abundances measured. For each of the stars in this list the $\chi^{2}$ of each chosen absorption line was measured for a range of $\log g$ 's, 3.5-5.0 dex in steps of 0.5. This allowed a wide parameter space to be probed and to test if the final metallicities were drastically affected by the surface gravity. It was found from $\chi^{2}$ fitting that the metallicity of these objects are only weakly dependent on the surface gravity and by interpolating between the points in $\chi^{2}$-space the error introduced is minimal.

However, the analysis in Sect. 5.3 requires some knowledge of the stellar surface gravity to use as a proxy for evolutionary status and this was done by searching the stellar spectra for a large region that would produce significant enough gravity sensitivity within our course grid of gravity models. The region of spectra chosen was centered at $6200 \AA$ and had a width of $200 \AA$. By extracting this region from all stellar spectra and comparing it to the same region extracted from the models, for each of the gravity values and best fit temperatures and metallicities, then the $\chi^{2}$ minimisation could be used to indicate the evolutionary status of the star. Since this was across a course grid in surface gravity space, only an indication could be made, and hence construction of a grid with higher resolution would be required to determine the actual surface gravity with any degree of certainty. However, a check of a number of objects that overlap Valenti \& Fischer (2005) and Gray (2005) have shown that the $\chi^{2}$ can determine the minimum and a selection can be performed to remove any low surface gravity objects. For instance, the star HD 27442 has been shown to have a $\log (g)$ of 3.78 by Valenti \& Fischer and 2.93 in Gray et al. and the $\chi^{2}$ here minimises at 3.5, which is the lowest gravity value in this study. This shows the technique can be used to select against too evolved and/or too young stars.

\subsubsection{Microturbulent velocity}

Another parameter that has been introduced to smooth over the differences obtained between the predicted and observed equivalent widths of absorption lines as a function of line strength is the microturbulence parameter $\left(v_{\text {mic }}\right)$. This arbitrary parameter is not necessarily required in 3D convective models of stellar atmospheres (Asplund et al. 2000) but has become a standard when dealing with one-dimensional stellar spectra to alleviate any discrepancies in equivalent width measurements. $v_{\text {mic }}$ is used to represent uncertainties in a number of parameters like excitation energy, oscillator strength, effective temperature, surface gravity etc. Valenti et al. find that $v_{\text {mic }}$ and the total stellar abundance $([\mathrm{M} / \mathrm{H}])$ are partially degenerate therefore they fixed the microturbulence velocity to $0.85 \mathrm{~km} \mathrm{~s}^{-1}$ to minimise errors in their final abundances. In this study the microturbulence parameter has already been fixed to $2 \mathrm{~km} \mathrm{~s}^{-1}$ as the Kurucz (1993) model atmospheres were computed for this value across all the required metallicity range and also to create a consistent sample. This does not introduce any significant uncertainty since our line list selection aimed to target weak lines that are formed deeper in the stellar interior and hence occupy the linear part of the curve of growth. Such lines are insensitive to changes in the microturbulent velocity. Hence, we believe that the final $\chi^{2}$ estimated error represents the uncertainty of this parameter. To fully test this we computed 10 values using microturbulences of both $1 \mathrm{~km} \mathrm{~s}^{-1}$ and $2 \mathrm{~km} \mathrm{~s}^{-1}$ for solar-type stars with a range of metallicities and found no offset of the final abundances, with only a small rms scatter of \pm 0.02 dex.

\subsubsection{Macroturbulent velocity and stellar rotation}

In the first approach the macroturbulent velocity $\left(v_{\mathrm{mac}}\right)$ parameter has the same affect on atomic line profiles as that of rotation or the instrumental profile, however it is described by a different profile than both these parameters. As it's name suggest, $v_{\text {mac }}$ is the term that considers the smaller scale motions of the stellar envelope induced by internal motions within the star. Highresolution spectra and detailed imaging of the Sun's surface that considers the larger scale motions has revealed velocity fields that vary typically at a few $\mathrm{km} \mathrm{s}^{-1}$ (e.g. Rieutord et al. 2001). These motions are generated by photospheric granulation and acoustic waves permeating throughout the star due to subsurface convection. This motion is also present in the high-resolution stellar spectra generated from the integrated flux from a stellar source and manifests itself by broadening line profiles. However, as the average resolution across the spectrum is $\sim 0.15 \AA$, (a velocity of $\sim 6 \mathrm{~km} \mathrm{~s}^{-1}$ ), we believe the broadening to be dominated by the stellar rotation as velocity fields of a few $\mathrm{km} \mathrm{s}^{-1}$ are comparable with the slowest rotation rates.

As mentioned above, stellar rotation $(v \sin i)$ has the effect of broadening line profiles without altering the line equivalent width, therefore knowledge of the rotational velocity allows a better representation of the line profile and will further reduce the uncertainty in the final metallicities. In order to determine the $v \sin i$ for each star we selected an iron line that was shown to 
exhibit little change in line strength through the range of metallicities. This line, which is centered at a wavelength of $7445.758 \AA$, is highlighted by an asterisk in Table 1 and is the strongest line in the list. The observed spectra around this line were then compared with the model spectra, broadened in steps of $1 \mathrm{~km} \mathrm{~s}^{-1}$ between $1-15 \mathrm{~km} \mathrm{~s}^{-1}$. We chose a width of $\pm 0.5 \AA$ centered on the line to compare each line with the models as this also included valuable wing information. $\chi^{2}$ values were generated at each velocity step and the minimum represents the final $v \sin i$. The resolution of the instrument at $7000 \AA$ is $\sim 6 \mathrm{~km} \mathrm{~s}^{-1}$, however we found that we can discern values of $v \sin i>2.5 \mathrm{~km} \mathrm{~s}^{-1}$.

\subsubsection{Model atmospheres}

To enable highly accurate chemical abundances to be determined from this dataset a number of utilities were used to generate relevant synthetic spectra to compare with the observations. The programme chosen to create the synthetic spectra was WITA6 (Pavlenko 2000). Moore (1956) was used for the atomic line list and damping constants, with the ATLAS 9 model atmospheres of Kurucz (1993) and oscillator strengths from Gurtovenko \& Kostik (1998).

The grid of Kurucz models range from $3500 \mathrm{~K}$ to $8000 \mathrm{~K}$ with surface gravities and metallicities from 3.5 to 5.0 and -1.5 to 1.0 respectively, ensuring coverage of almost all possible values for these parameters. A few objects require lower metallicity models to accurately measure their values. A parameter that is fixed throughout is the microturbulent velocity. This is held fixed at $2 \mathrm{~km} \mathrm{~s}^{-1}$ for all the model grids, which will introduce some error into the accuracy of the final synthetic spectrum but after investigation it was found to be small compared with other uncertainties.

Since most of our objects are G and K type stars these models are adequate for metallicity computation, however Smalley \& Kupka (1997) have shown that there are errors inherent in them due to an inadequate convective overshooting algorithm. Although the errors are small for $\mathrm{G}$ and $\mathrm{K}$ stars, they can be significant for hotter $\mathrm{F}$ type stars (Gray et al. 2003). To investigate this we compute the metallicities of all objects with effective temperatures greater than $6000 \mathrm{~K}$, with and without convective overshooting. The results from the convective overshooting OnOff returned a mean of $-0.01 \pm 0.06$ dex for a total of 19 data points, which is consistent with an additional scatter of $0.06 \mathrm{dex}$ for the hottest stars in this sample.

\subsubsection{Line selection}

One of the most critical parts of abundance determination is the selection of lines to use in the observed spectrum to obtain a clean line profile. A vast number of the lines in a star such as the Sun are blended, especially towards the bluer end of the visual band (see Fig. 4 upper panel). The lines used in this work have been selected because they appear unblended at this spectral resolution (Fig. 4 lower panel) and in general they are also weak. Weak lines lie on the linear part of the curve of growth, therefore they are more sensitive to large changes in the model parameters and are better indicators of line profile characteristics giving more accurate abundances. A line's position on the curve of growth will significantly affect all parameters associated with the line as the curve of growth determines how the line evolves when more absorbers are introduced. For example, optically thick lines will saturate more quickly and therefore are not good estimators of the line strength and abundance status of
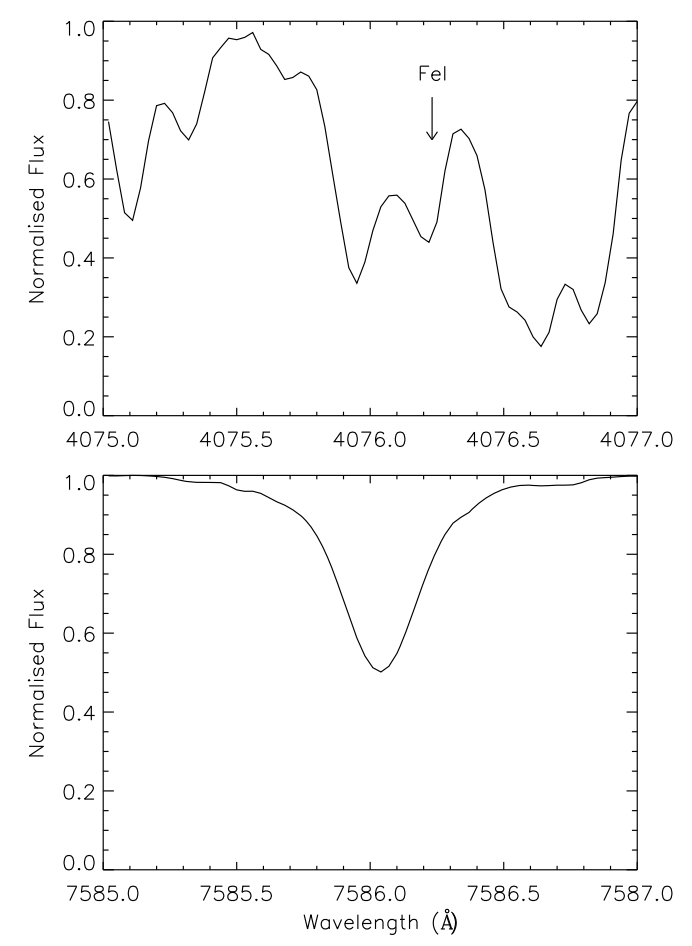

Fig. 4. A spectrum of the star HD 38459 towards the bluer end of the stars visual band is shown in the upper panel. All of the lines in this wavelength region (4074-4078 $\AA$ ) are blended. The FeI line in the middle of this region (4076.22 $\AA$ ) is clearly blended at both sides. The lower panel shows the iron line at $7586.027 \AA$. No strong line blends can be discerned from the noise at this spectral resolution and if any do exist they are rather marginal.

the star. Strong lines also add extra uncertainty through saturation, microturbulence and uncertainties associated with damping constants (e.g. see Gray 2005).

The FEROS spectra used here have a $\mathrm{S} / \mathrm{N}$ and resolution sufficiently high to allow a large number of apparently unblended lines to be used in this analysis. The lower wavelength cutoff chosen was at a $\lambda>5400 \AA$, this was due to the increased blending factor mentioned above (Fig. 4), however any lines below $6500 \AA$ were very carefully selected to ensure they were isolated from other possible blends. The wide coverage obtained using FEROS ( 3500-9200 ̊ Kaufer et al. 1999) allows one to select a large enough number of unblended iron lines. Solar spectra from FEROS, taken as part of the standard ESO Calibration plan, was used as a template to select lines that would appear in all other spectra. Also, no telluric lines blends were seen. All lines used for abundance determination, along with their wavelengths, oscillator strengths and excitation energies are shown in Table 1 . The line highlighted by the asterisk was used for the $v \sin i$ determination.

\subsubsection{Oscillator strengths}

One of the larger sources of error comes from uncertainties in the oscillator strengths. The chosen oscillator strengths were extracted from the list of Gurtovenko \& Kostik (1998). They determine the oscillator strength $(g f)$ using measurements of the equivalent widths, central intensities along with the synthesis of the whole Fraunhofer spectrum of the specific chemical element. They define two separate measurements of $g f$ using the equivalent widths of the lines and the central line depths to 
Table 1. Fe lines used in the abundance measurements.

\begin{tabular}{|c|c|c|c|}
\hline Element & $\lambda(\AA)$ & $g f$ & $E(\mathrm{keV})$ \\
\hline FeI & 5806.723 & $1.096 \mathrm{E}-01$ & 4.610 \\
\hline FeI & 5852.215 & $5.623 \mathrm{E}-02$ & 4.550 \\
\hline FeI & 5855.082 & $2.239 \mathrm{E}-02$ & 4.610 \\
\hline FeI & 5856.086 & $2.291 \mathrm{E}-02$ & 4.290 \\
\hline $\mathrm{FeI}$ & 6027.051 & $5.888 \mathrm{E}-02$ & 4.070 \\
\hline FeI & 6151.621 & $4.266 \mathrm{E}-04$ & 2.180 \\
\hline FeI & 6159.379 & $1.148 \mathrm{E}-02$ & 4.610 \\
\hline FeI & 6165.359 & $2.754 \mathrm{E}-02$ & 4.140 \\
\hline FeI & 6173.340 & $1.259 \mathrm{E}-03$ & 2.220 \\
\hline FeI & 6229.227 & $9.120 \mathrm{E}-04$ & 2.840 \\
\hline FeI & 6608.027 & $9.120 \mathrm{E}-05$ & 2.280 \\
\hline FeI & 6627.547 & $2.570 \mathrm{E}-02$ & 4.550 \\
\hline FeI & 6703.566 & $8.318 \mathrm{E}-04$ & 2.760 \\
\hline FeI & 6725.359 & $5.370 \mathrm{E}-03$ & 4.100 \\
\hline FeI & 6750.152 & $2.344 \mathrm{E}-03$ & 2.420 \\
\hline FeI & 6810.266 & $8.710 \mathrm{E}-02$ & 4.610 \\
\hline FeI & 7158.477 & $1.148 \mathrm{E}-03$ & 3.650 \\
\hline FeI & 7189.152 & $1.585 \mathrm{E}-03$ & 3.070 \\
\hline $\mathrm{FeI}^{*}$ & 7445.758 & $6.761 \mathrm{E}-01$ & 4.260 \\
\hline FeI & 7491.656 & $8.710 \mathrm{E}-02$ & 4.300 \\
\hline FeI & 7568.906 & $1.288 \mathrm{E}-01$ & 4.280 \\
\hline FeI & 7586.023 & $7.079 \mathrm{E}-01$ & 4.310 \\
\hline FeI & 7751.109 & $1.514 \mathrm{E}-01$ & 4.990 \\
\hline FeI & 7780.562 & $1.148 \mathrm{E}+00$ & 4.470 \\
\hline FeI & 7807.914 & $2.754 \mathrm{E}-01$ & 4.990 \\
\hline FeI & 7832.207 & $1.259 \mathrm{E}+00$ & 4.430 \\
\hline FeI & 8047.625 & $2.239 \mathrm{E}-05$ & 0.860 \\
\hline FeI & 8239.137 & $3.715 \mathrm{E}-04$ & 2.420 \\
\hline
\end{tabular}

allow them to estimate the accuracy of the values and to generate estimates of the uncertainties in the data. They found that the internal accuracy of the final $\log (g f)$ values to typically be \pm 0.07 dex. Recently Bigot \& Thévenin (2006) have determined the oscillator strengths for FeI and SiI in the Gaia spectral window ( $~ 8480-8750 \AA)$ and found accuracies of typically $<0.1$ dex with laboratory results, whereas the oscillator strength accuracies found in databases such as the Vienna Atomic Line Database can be much lower.

\subsubsection{WITA program}

The latest version in the WITA series of programs was used to generate the grid of synthetic spectra. The WITA series were developed as part of the ABEL6 complex (Pavlenko 1991), which was composed partly from Kurucz (1979) ATLAS subprograms. The WITA6 programme (Pavlenko 2000) itself is a modified version of the WITA2 programme (Pavlenko et al. 1995). All computations performed by WITA6 are employed in the classical way, using Local Thermodynamical Equilibrium (LTE) and assuming hydrodynamic equilibrium for one-dimensional model atmospheres. The code allows for the extrapolation of the model atmosphere. The ionisation-dissociation equilibria equations system has been solved for various temperature structures assuming LTE. A Voigt profile was adopted for single absorption lines, taking into consideration all line broadening sources, such as natural broadening, thermal broadening, pressure broadening and resonance broadening.

\subsubsection{Continuum normalisation}

No flux calibration was performed which left behind a gradient in the spectral profile across the measured wavelength region.
To attempt to detect the true continuum level around each line a cubic-spline fit procedure was performed using the programme CONTINUUM part of the IRAF library of routines. A global fit was used across the entire wavelength region. By employing tight constraints on the upper and lower limit rejection thresholds of the fitted data points a good continuum measurement was made for the entire spectra.

\section{4. $\chi^{2}$ fitting}

Once all the parameters of each object have been fit and iron lines selected the final step is to generate the desired $[\mathrm{Fe} / \mathrm{H}]$ abundance. An IDL code has been developed that will employ several different steps to compare the lines in the real spectrum with the corresponding lines in the synthetic spectrum and generate a $\chi^{2}$ value for each line in each star. These $\chi^{2}$ 's will minimise when the synthetic spectrum best replicates the real spectrum, allowing $[\mathrm{Fe} / \mathrm{H}]$ abundances to be determined.

The first step in the programme takes the generated temperature values of each star and then selects the spectra that have the nearest temperatures above and below the generated temperature in the grid. The Kurucz grid has temperature steps of $250 \mathrm{~K}$, therefore to better replicate the real spectra the upper and lower spectra are interpolated to an improved temperature scale. It is at this stage when the synthetic spectrum is broadened for stellar rotation and the instrumental profile. The rotation rates are generated following the procedure described above. The instrumental profile is modeled by convolution with a Gaussian profile of width determined by the resolution of FEROS. The FEROS resolution is $\sim 48000$, which at these wavelengths corresponds to a Gaussian of width $\sim 0.15 \AA$ however since the instrumental profile changes with wavelength (e.g. telluric lines in the far red of the chip have widths $>0.2 \AA$ ) we also use the models to better define an optimum broadening width.

$\chi^{2}=\frac{\left(O^{2}-M^{2}\right)}{\sigma^{2}}$

After the model spectra have been prepared for comparison with the real spectrum the programme selects each individual line that has been selected for comparison and employs a $\chi^{2}$ fit between the real and model spectra. Equation (2) describes the $\chi^{2}$ employed for this fitting procedure, where $O$ is the observed spectra, $M$ is the modeled spectra and $\sigma$ is the total errors in both. This $\chi^{2}$ equation is applied to each line by selecting the line centre and comparing a region $\pm 0.2 \AA$ from the center. The $0.4 \AA$ window was selected by eye after scrutinising all lines and finding the optimum window for these lines in the FEROS spectra. As this window is only an optimal window and will include more continuum in some lines than others, the median of all the $\chi^{2}$ values is used. This helps remove any outlying values due to bad lines, any possible small edge blending and extraction errors. The median is generated for each metallicity value four times, representing the four different $\log (g)$ 's for each metallicity. Figure 5 shows the fitting output of the star HD 1835. The crosses are the medians of the four separate $\log (g)$ fits and the solid line represents the bestfit second-order polynomial to the points. The minimum of this function represents the stars $[\mathrm{Fe} / \mathrm{H}]$ abundance, which for this star is $0.21 \pm 0.03$, agreeing with the Valenti \& Fischer (2005) value of $0.25 \pm 0.03$. This entire procedure is applied to all targets. 


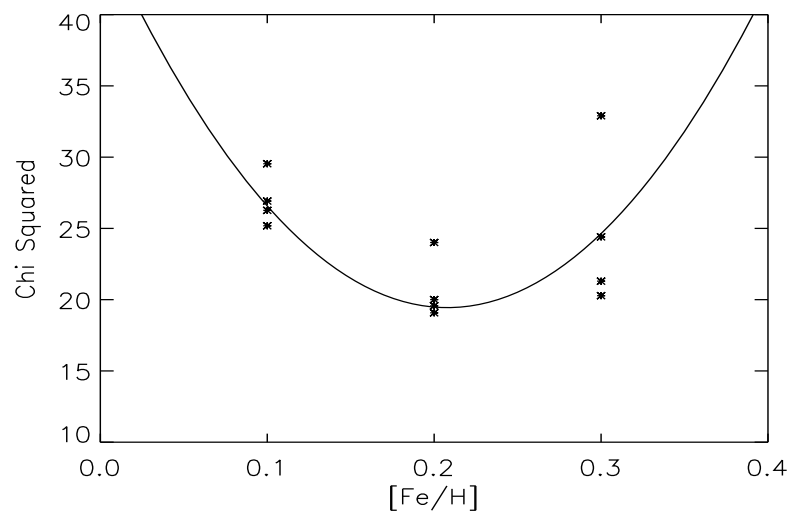

Fig. 5. The $\chi^{2}$ fit for HD 1835 in metallicity space. The points represent the means of four $\chi^{2}$ values for different surface gravities at each of these metallicities. The solid line is the second-order polynomial fit to the points, with the final metallicity representing the minimum of this function.

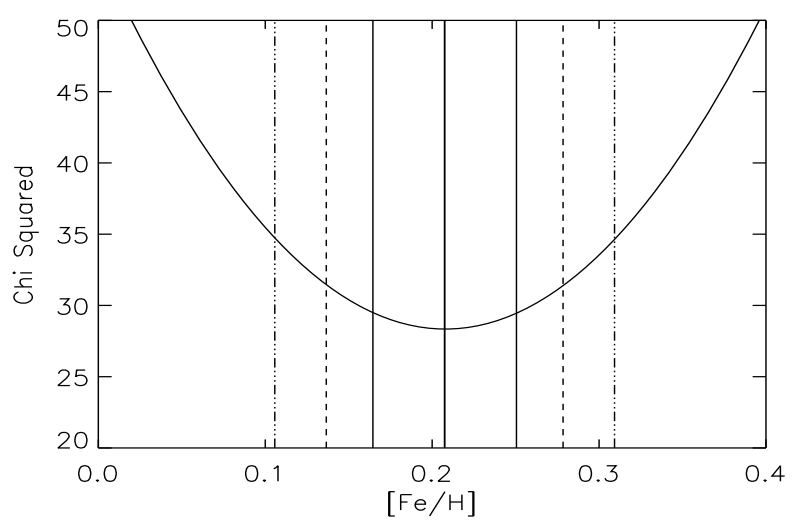

Fig. 6. The $\chi^{2}$ fit across all metallicity values of the star HD 1835 with the $1 \sigma$ (solid bounds), $2 \sigma$ (dashed bounds) and $3 \sigma$ (dot-dashed bounds) error estimates shown. These errors ranges were selected by interpolating a table of $\chi^{2}$ steps for a two parameter fit i.e. surface gravity and metallicity. The central solid line marks the minimum of the fitted function and represents the final abundance value derived for this star.

\subsection{1. $\chi^{2}$ uncertainties}

To determine the errors in the final abundance measurements the fitted $\chi^{2}$ parabola was used to interpolate the 1,2 and $3 \sigma$ values using a table of $\chi^{2}$ values. Figure 6 shows the $\chi^{2}$ space for all three of these error ranges. The solid lines bound the $1 \sigma$ error region, with the central solid line marking the minimum of the function or the final abundance measurement. The dashed and dot-dashed regions mark the 2 and $3 \sigma$ parameter space. Since the function is fit to both the $\log (g)$ and the iron abundance a two parameter $\chi^{2}$ error step was performed. The $1 \sigma$ errors have a step of 1.155 from the minimum value, with the 2 and $3 \sigma$ steps equaling 3.118 and 6.396 respectively. This error analysis is only valid assuming one has a handle on the uncertainty, however as shown before their are a number of uncertainty sources that are difficult to quantify, (i.e. atomic oscillator strengths), therefore the final $\chi^{2}$ curve must represent this uncertainty. To accomplish this the reduced Chi-squared $\left(\chi_{r}^{2}\right)$ was also determined by dividing the $\chi^{2}$ by the number of degrees of freedom. The degrees of freedom were found by subtracting the number of fitted parameters from the number of data points of each iron line. Ideally the $\chi_{r}^{2}$ should equal, or be close to 1 for a good fit with all uncertainty sources well modeled. However, since many uncertainties are poorly constrained, the $\chi^{2}$ function was normalised by the

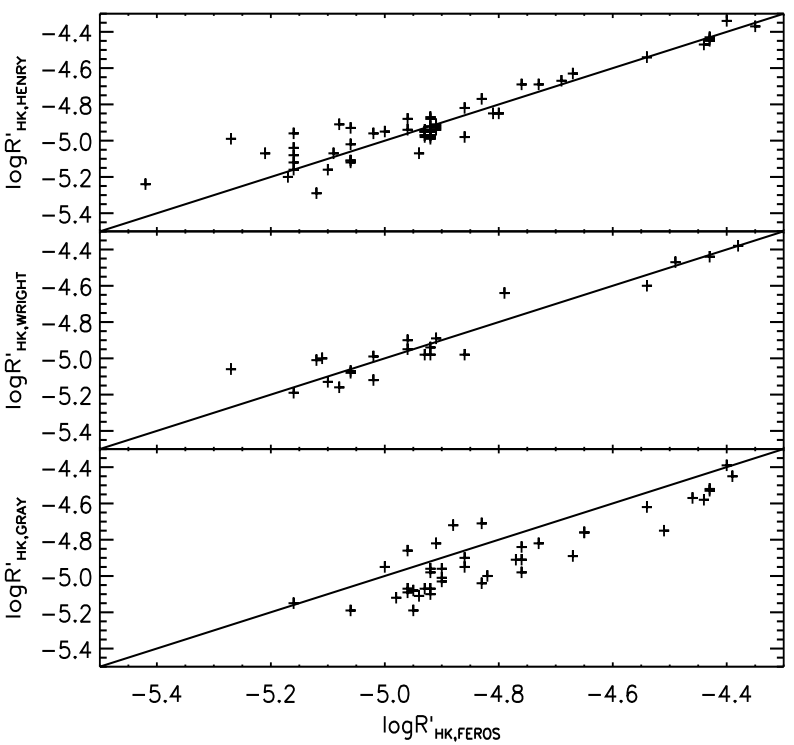

Fig. 7. A direct comparison of $\log R_{H K}^{\prime}$ values from this work against Henry et al. (1996) (upper), Wright (2004a) (middle) and Gray et al. (2006) (lower). The plots show good agreement with Henry et al. and Wright et al. and both have rms scatters of $\sim 0.08$ in log space. However, there appears a small offset with the results of Gray et al., with the values here slightly higher by around $0.1 \mathrm{dex}$. The solid line through the data represent a 1:1 relationship.

difference between the minimum of the $\chi_{r}^{2}$ function and the optimum (i.e. 1). This has the same effect as randomly adding percentage errors into the original $\chi^{2}$ equation (Eq. (2)) to broaden the fitting function and increase the final uncertainty estimates of all abundances.

\section{Comparison with previous work}

\subsection{Activities}

To make judgments and comparisons of this data to other published work we have plotted the individual activity values against those of three other catalogues. Figure 7 shows the comparisons of these values to those of Henry et al. (1996) (upper panel), Wright (2004a) (middle panel) and Gray et al. (2006) (lower panel). The comparison to that of the Henry et al. values shows a scatter around the 1:1 relation (solid line) with no apparent offsets. Considering they also used calibrators from Duncan et al. (1991) one would expect there to be a good relationship. However, the Gray et al. work makes use of low-resolution spectra and also uses a different setup from that employed here. They centered $4 \AA$ square bandpasses on the CaII HK line cores instead of triangular bandpasses of width $1.09 \AA$ which is used at Mt. Wilson and also used here. This will contribute to an increase in the scatter between the data and also to non-linear calibrations onto the MW system. The rms scatter in this data is found to be $\sim 0.08$. A Student-T test was performed on all three of these distributions to test if they have the same true means. The test revealed an $83 \%$ probability that the data from here and that of Henry et al. are drawn from the same mean, which given the intrinsic variability associated with cool dwarf stars and the limited number of data points is noteworthy.

The middle panel in Fig. 7 shows the data from Wright (2004a) against the values found here. There appears a good fit to this data around the $1: 1$ relation with only two outliers. This is a nice fit when considering the intrinsic scatter inherent in the 


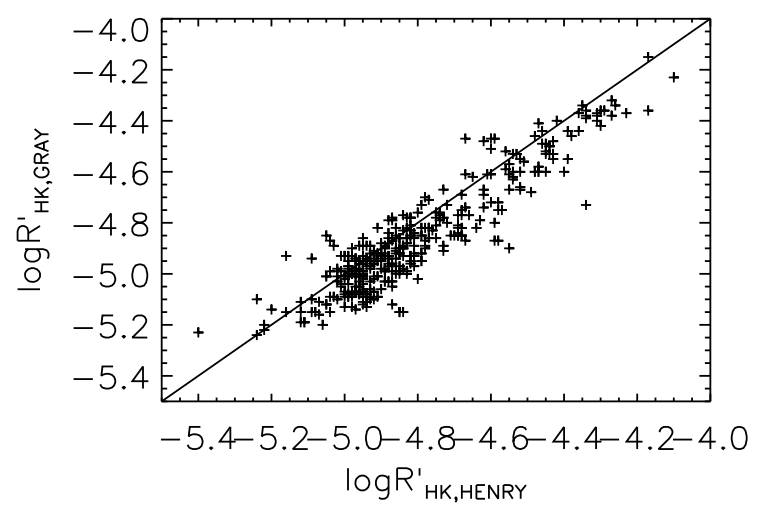

Fig. 8. A comparison of the $\log R_{H K}^{\prime}$ activity indices from Henry et al. (1996) and Gray et al. (2006). There is an offset in the data, with the values of Gray et al. around 0.1 dex lower than those of Henry et al.

values themselves, which can alter by 0.15 in $\log R_{H K}^{\prime}$ over a period of a few years. The rms scatter here is $\sim 0.08$, the same as the scatter of Henry et al. However, the two outlying stars (HD 8038 and HD 150437) cause this unweighted rms to deviate from the true scatter. Once these two stars have been removed the rms scatter is found to be 0.06 , highlighting the close relationship that is found using high-resolution spectra and a setup similar to that of the MW project. The T test of this data reveal that they are both drawn from the same mean at a probability of $88 \%$, which is high given the limited number of data points.

A recent large survey in the southern hemisphere has been conducted by Gray et al. (2006) connected to the NStars Project. The lower panel in Fig. 7 compares our measurements to this data. There is some scatter around the 1:1 relation, however the majority of objects lie below this fit indicating a possible systematic offset in the data. Neglecting the stars above the 1:1 fit there is an offset of around -0.1 in $\log R_{H K}^{\prime}$ that is not present in both other studies. A straight line fit to describe the offset has a gradient of $0.938 \pm 0.006$ and offset of $-0.389 \pm 0.362$, with an rms scatter of 0.096 . This can be used to transform these results to the scale of Gray et al. The significance of the offset is at the $2 \sigma$ level as the $\mathrm{T}$ test of this comparison gave a probability of $96 \%$ that both distributions were drawn from different true means. We also performed the $\log R_{H K}^{\prime}$ conversion including all the stars in common with Gray et al., however once this calibration has been performed, both Henry et al. and Wright are offset from this work by around +0.1 dex. The point can be highlighted by plotting Gray et al. against the work of Henry et al. as both studies used similar resolutions and setups (Fig. 8). It can be seen from this comparison that the Gray et al. dataset is around 0.1 dex lower in $\log R_{H K}^{\prime}$, which is difficult to explain by intrinsic scatter. Indeed, the $T$ test revealed a probability of $98 \%$ that both have been drawn from different true means. The offsets could arise due to the different calibrators used by this and the Henry et al. study against those of Gray et al. Gray et al. used calibrating stars from Baliunas et al. (1995) compared to the Duncan et al. values, however as no offset appears between this study and Wright, (who also calibrated from Baliunas et al.), this seems unlikely. It may simply arise from a combination of the calibrators and the wide bandpasses used to extract the activities as the width, shape and resolution in the line cores can serve to mask any emission feature and hence mask the true activity of a star. Note also that Henry et al. performed non-linear calibrations onto the MW system, whereas both this work and Gray et al. use linear calibrations and this may explain some of the offsets seen, especially towards the more active end of the calibration. Whatever the cause it is clear a more thorough look at all these studies together, along with long term variability data is needed to extract a good list of intrinsically stable stars to use as calibrators for similar studies in the southern hemisphere.

\subsection{Metallicity}

An integral part of any abundance determination of a large sample of solar-type stars is to compare the final values with those published in other work. We have chosen three of the most recent large bodies of published metallicity values in the southern hemisphere to compare against our determined abundances. The three works we have compared against are Valenti \& Fischer (2005), Nordström et al. (2004) and Gray et al. (2006). The most appropriate work to compare the results found here with are those of Valenti \& Fischer as the methodologies are similar. They determine their final abundances by directly comparing observed high-resolution, high-S/N echelle spectra against a grid of synthetic model spectra generated using Kurucz (1992) model atmospheres. This comparison is shown for both the effective temperatures and the metallicities in the upper panels (left and right) of Fig. 9. The $T_{\mathrm{EFF}, V a l e n t i}$ and $[\mathrm{Fe} / \mathrm{H}]_{\text {Valenti }}$ are the effective temperatures and iron abundances by Valenti \& Fischer and the $T_{\text {EFF,FEROS }}$ and $[\mathrm{Fe} / \mathrm{H}]_{\text {FEROS }}$ are similar but for the values found in this project. The solid lines represent a 1:1 relationship and it is clear that the values in both panels are in good agreement. The effective temperatures (left panel) in Valenti \& Fischer were derived by a spectral fitting procedure, which is different to our photometrically derived IRFM values, however the data are in good agreement with only two outliers that are significantly below the 1:1 relationship. Both outliers have been found to exhibit some level of variability by Hipparcos and hence the photometric method can produce spurious results. The rms scatter around a linear fit to this data is $\pm 132 \mathrm{~K}$, which reduces to $\pm 80 \mathrm{~K}$ once the two variable outliers have been removed. The Student-T test reveals a $85 \%$ probability that both these have similar means, increasing to $91 \%$ when the outliers have been removed. The metallicity comparison (right panel) shows a tight relationship between that data. This is confirmed by the scatter of only \pm 0.09 , reducing to \pm 0.05 once the two outliers have been removed. The T-test revealed a $96 \%$ probability that both distributions have the same means, reducing to $95 \%$ when the outliers have been removed. However, if we consider all objects with metallicities $\geq 0.1$ dex from both Valenti \& Fischer and here, then the Valenti $\&$ Fischer values are found to be more metal-rich by $+0.025 \mathrm{dex}$.

The middle and lower panels in Fig. 9 show comparisons against the two other southern samples, however the methodologies were different to that employed here. The middle panel shows the comparison with the values published in Nordström et al. (2004) $\left([\mathrm{M} / \mathrm{H}]_{\text {Nordstrom }}\right)$. Nordström et al. determined the metallicity of $\sim 14000$ stars using relationships between the overall stellar metallicity and their Strömgren colours. Strömgren photometry has proven a useful technique in estimating a star's metallicity, however the calibrations tend to have larger systematic uncertainty than spectroscopically determined abundances due to a lack of calibrators, particularly towards the reddest stars studied (e.g. Twarog et al. 2002). Nordström et al. created their own calibrations following techniques previously employed by other authors (e.g., Schuster \& Nissen 1989; Edvardsson et al. 1993) and they find a scatter of 0.12 for $G$ and K-type stars, reducing to 0.1 for F-types. It must be noted that the relations determined are compared to the iron abundances $([\mathrm{Fe} / \mathrm{H}])$ of the calibrator stars since iron is an accurate tracer of the overall stellar metallicity abundance. From Fig. 9 

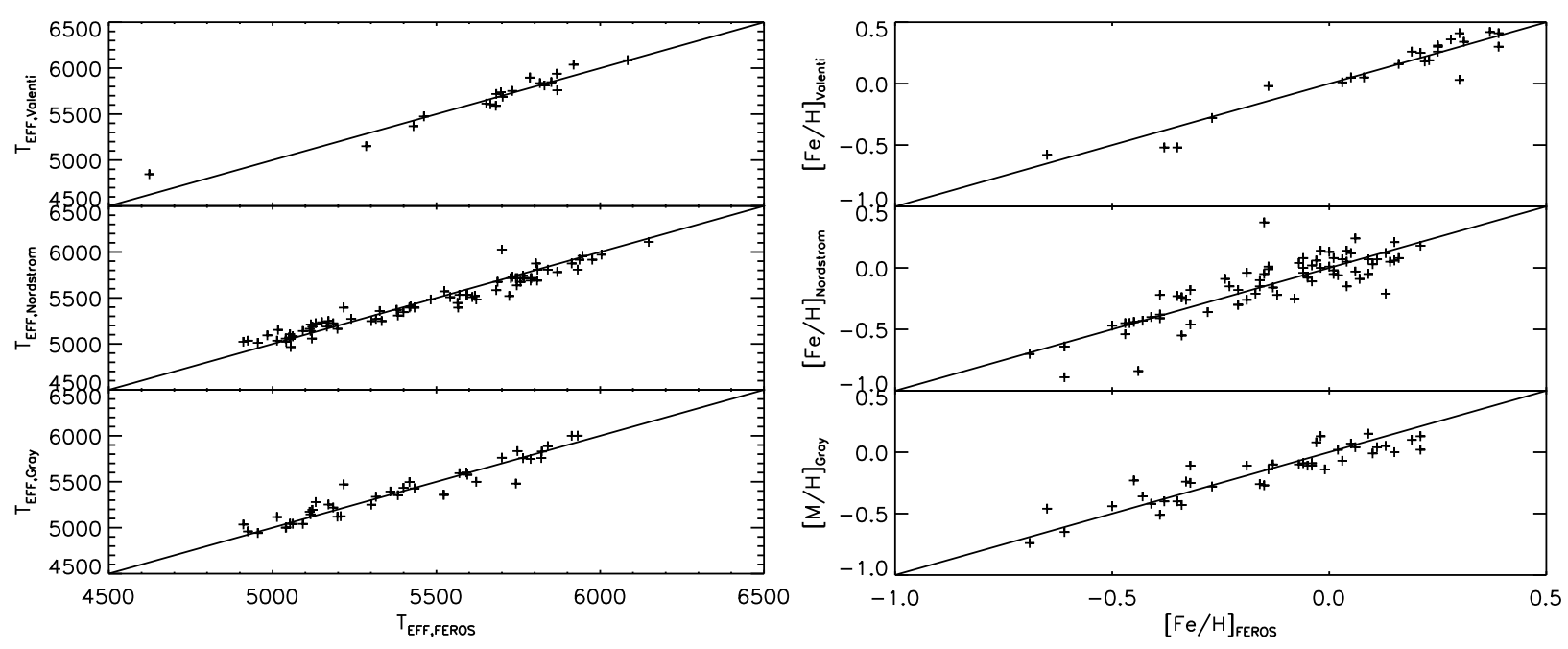

Fig. 9. A direct comparison of effective temperatures (left panels) and metallicity values (right panels) determined here and those published in recent work. The upper plots are a comparison to the values published in Valenti \& Fischer (2005), which were derived using spectral line fitting similar to the analysis in this project for metallicities but differing in effective temperature determination. The middle plots are a comparison with the values of Nordström et al. (2004), which were derived from Strömgren photometry. The lower plots compare against the values published in Gray et al. (2006), derived by fitting to low-resolution spectra. The effective temperatures agree well with those of Valenti \& Fischer, with only two outliers that are variable stars. There appears to be offsets between this work and both Nordström et al. and Gray et al. The metallicity plots agree well with all three of the published work, with the lowest rms scatter found against the work of Valenti \& Fischer due to the similarity in the methodology. However, a scatter of only $\sim 0.1$ was also found compared to the other methods. The solid lines through all six plots represent the $1: 1$ relationship.

(middle left) it can be seen that the scatter in effective temperature between this work and Nordström et al. is lower than both the other two works. The measured rms scatter is $73 \mathrm{~K}$, however a step change of $\pm 50 \mathrm{~K}$ seems apparent at an effective temperature of $5250 \mathrm{~K}$. For the whole sample the T-test reveals a $82 \%$ probability that both these distributions have similar means. However, for the lower and upper temperature cut the probabilities are $5 \%$ and $28 \%$ respectively. These offsets mainly arise due to the different methodologies used to generate the effective temperatures i.e. Strömgren versus Johnson filters. The metallicity comparison (middle right) has a scatter of 0.13 dex and a T-test probability of $100 \%$ that both are drawn from the same mean. This level of agreement between the two data show that with a sufficiently large comparison dataset, the values in this work are as accurate as any measurements currently in the literature.

As mentioned in Sect. 4.1, the Gray et al. work is part of the NStars Project and their methodology employed fitting models directly to a large spectral region of low-resolution spectra, whilst also fitting to the observed and synthetic fluxes (see Gray et al. 2003). The synthetic spectra were generated using Kurucz (1993) model atmospheres and the spectral synthesis programme SPECTRUM. A chi-squared minimisation technique was used, in conjunction with a SIMPLEX interpolation to generate a global fit to the observed spectra. Again Gray et al. provides a useful test as their method made use of stellar spectra to fit models directly. They have also compared their overall $[\mathrm{M} / \mathrm{H}]$ values to the $[\mathrm{Fe} / \mathrm{H}]$ abundances measured by Valenti \& Fischer (2005) and found a scatter of only 0.09 dex, however they found an offset of -0.07 dex. From the lower right plot in Fig. 9 it can again be seen that the derived metallicity values are in good agreement with those of Gray et al. $\left([\mathrm{M} / \mathrm{H}]_{\text {Gray }}\right)$. The scatter is found to be $0.09 \mathrm{dex}$, which compares well with the scatter found between Gray et al. and Valenti \& Fischer. There may be an offset of -0.01 dex between these samples, however since this is below the general scatter in the data, this is deemed negligible. This is confirmed by the T-test which reveals a probability of
$81 \%$, indicating that the offset is not significant. The lower left plot in Fig. 9 shows the comparison with the Gray et al. effective temperatures and a good correlation is found between the two datasets. The scatter is found to be $\pm 90 \mathrm{~K}$ and no significant offset was found, giving rise to a probability of $93 \%$ that both datasets have the same means. Since Gray et al. had shown their values to be in agreement with those of the IRFM, a good correlation was to be expected. By comparing FEROS values to three independent studies that employed three different methodologies it is clear that these values provide a robust assessment of the effective temperatures and iron abundances of all stars in this sample.

\section{Results and discussion}

\subsection{Activity analysis}

The distribution of chromospheric activity of solar-type stars has been shown to follow a bimodal distribution with both an active and inactive peak (e.g. Duncan et al. 1991; Henry et al. 1996; Gray et al. 2006; J06). The upper panel in Fig. 10 shows the distribution of stellar $\log R_{H K}^{\prime}$ in this study and confirms the bimodal peaks found before. The plot has been binned up with bin widths of 0.05 in $\log R_{H K}^{\prime}$ and both peaks are easily identifiable. A bimodal gaussian has been fit to the data and is modelled by the dashed line in the plot. The peaks of this distribution are centered at -4.52 (active) and -5.00 (inactive) and have standard deviations of 0.13 and 0.10 respectively. There are a few stars out in the tails of the distribution. HD 27442 and HD 147873 are located out in the inactive wing and have $\log R_{H K}^{\prime}$ indices of -5.39 and -5.42 respectively.

The lower plot in Fig. 10 shows the residuals of the double Gaussian fit to the distribution. The stars located out in the active tail of the fit are single objects and therefore the residual errors bars will place them in agreement with the fit as the uncertainty is approximated using Poisson root-N, statistics. From these statistics alone it is hard to test whether or not these stars 


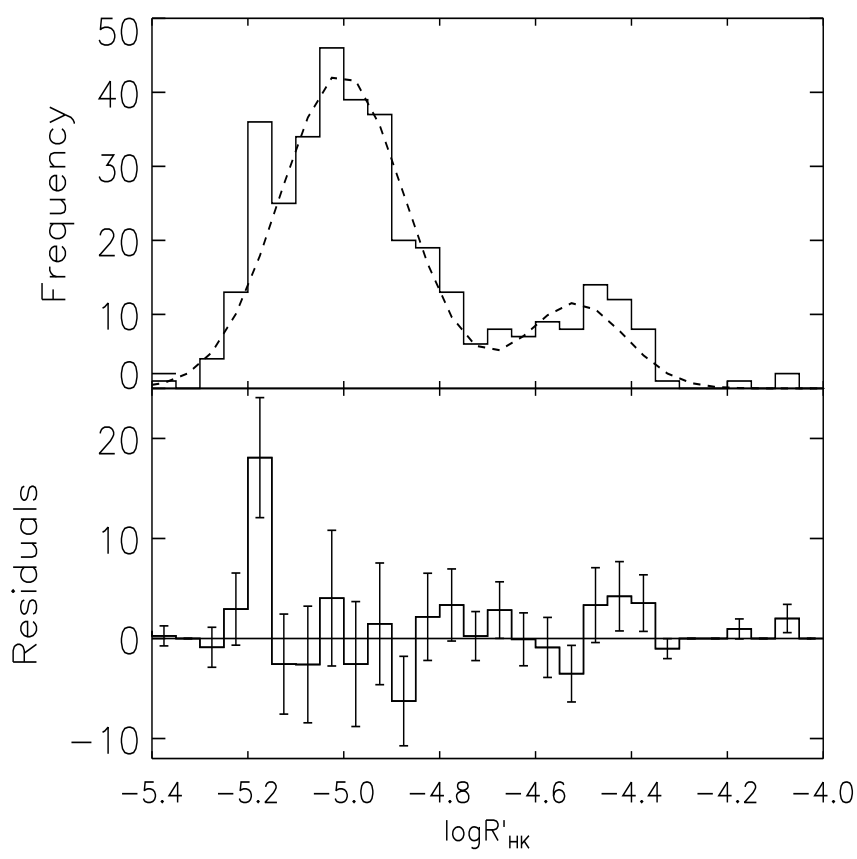

Fig. 10. The upper plot shows the $\log R_{H K}^{\prime}$ distribution of all bright stars in the southern hemisphere in this study and the inactive and active peaks are confirmed. The overall distribution is in agreement with the bimodal fits from Henry et al. (1996) and Gray et al. (2006) in the southern hemisphere, however a third component may exist. The best-fit to the data employs a bimodal distribution and this is overplotted by the dashed line. The means of the Gaussians are -4.52 (active) and -5.00 (inactive) with standard deviations of 0.13 and 0.10 respectively. The lower plot shows the residuals of the fit along with their associated error bars. Most of the bins are in good agreement with the fit, with only one bin over $2 \sigma$ away from the fit. This bin contains mostly stars evolving off the main sequence.

are undergoing an active or inactive phase of their evolution. Almost all the bins agree well with the fit within the uncertainties shown except for one which is over $2 \sigma$ away from the fit. It will be shown later in the chapter that this bin, which is centered at -5.175 , contains mostly evolved stars. It is interesting to note that this result was also found by Gray et al. (2003) at around the same inactive bin of their distribution. They concluded that this may partly be caused by a large number of Maunder minimum stars. However, Wright (2004b) found that most stars announced as in Maunder minimum phases were in fact evolving off the main sequence and hence the activity methodology used to measure their indices are not entirely adequate for comparison with main sequence stars. A trimodal fit was also applied to the data to test if the fit was significantly improved. The $\chi^{2}$ of the bimodal distribution was 30 with 23 degrees of freedom, compared with the value of 21 with 20 degrees of freedom for the trimodal distribution. Therefore the reduced $\chi^{2}$ values are 1.29 and 1.06 respectively. Thus, it is not clear that a significant improvement is made by assuming a third peak exists in the data.

\subsection{Metallicity analysis}

To assess the validity of the metallicity abundances of each of the target stars a check of line excitation energy and wavelength dependency is performed. The final abundance for each line is computed following the same procedure as above. This abundance is then subtracted from each star's total abundance and plotted against both excitation energy and wavelength of the given line. Figure 11 shows both the excitation energy (upper
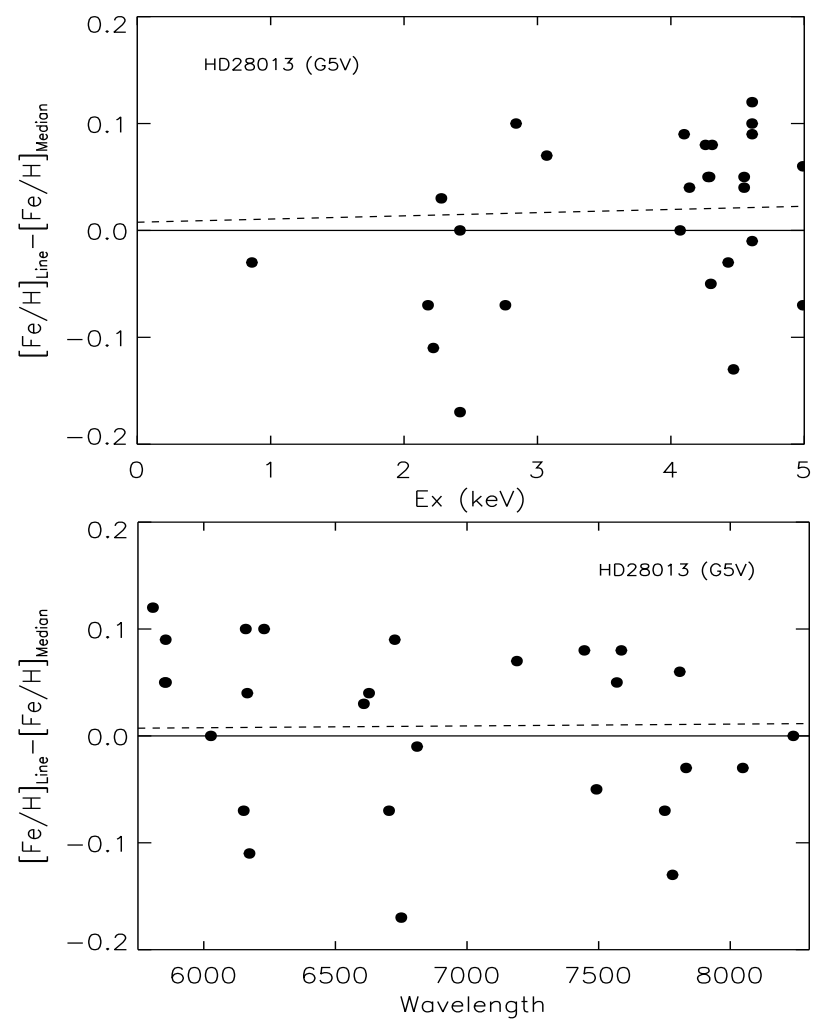

Fig. 11. A check of the dependency of the final abundance measurements on both absorption line excitation energy (upper panel) and wavelength (lower panel) of the star HD 28013. These plots are typical of the whole sample. This shows that the final abundance values are not significantly dependent on excitation energy or wavelength. The dashed lines represent best fit linear trends to the data. The solid line marks the zero points.

panel) and wavelength (lower panel) abundances for each of the lines chosen here in the solar analogue (Spectral type G5V; $[\mathrm{Fe} / \mathrm{H}]=0.01) \mathrm{HD} 28013$. The majority of data points are spread within \pm 0.1 dex from the final abundance value in both plots, which is represented by the solid line, and it is these clustered points that give rise to the measured abundance value. A linear least-squares fit was applied to the data and these are represented by the dashed lines in both plots. It is clear that the gradient of these lines are very small, indicating that there is very little dependence of the abundance measurements on excitation energy or wavelength. These plots are typical of the whole sample of objects and indicate that the computed $[\mathrm{Fe} / \mathrm{H}]$ abundances are reliable and not a function of the selected lines.

The overall distribution of metallicities for all stars in this sample is shown in the upper panel of Fig. 12. The peak of the distribution resides at $\sim 0.0-0.1 \mathrm{dex}$, however it is hard to draw any inferences about the distribution of metallicities in the solar neighbourhood as there are specific biases in the data. For instance, a subsection of the data had Strömgren colours measured by Hauck \& Mermilliod (1998) and using calibrations found in Haywood (2002) we were able to obtain a first approximation of the overall metallicity of these objects. We then selected a subset of the most metal-rich of these objects to follow-up with spectroscopy to confirm their metal-rich status and further boost the numbers of metal-rich objects that will constitute the final target list. However, as explained in Sect. 3.3, the primary list of targets were objects with no known high-resolution metallicity measurements, to further enhance the database of fundamental stellar parameters in the southern hemisphere. Also shown in this figure 


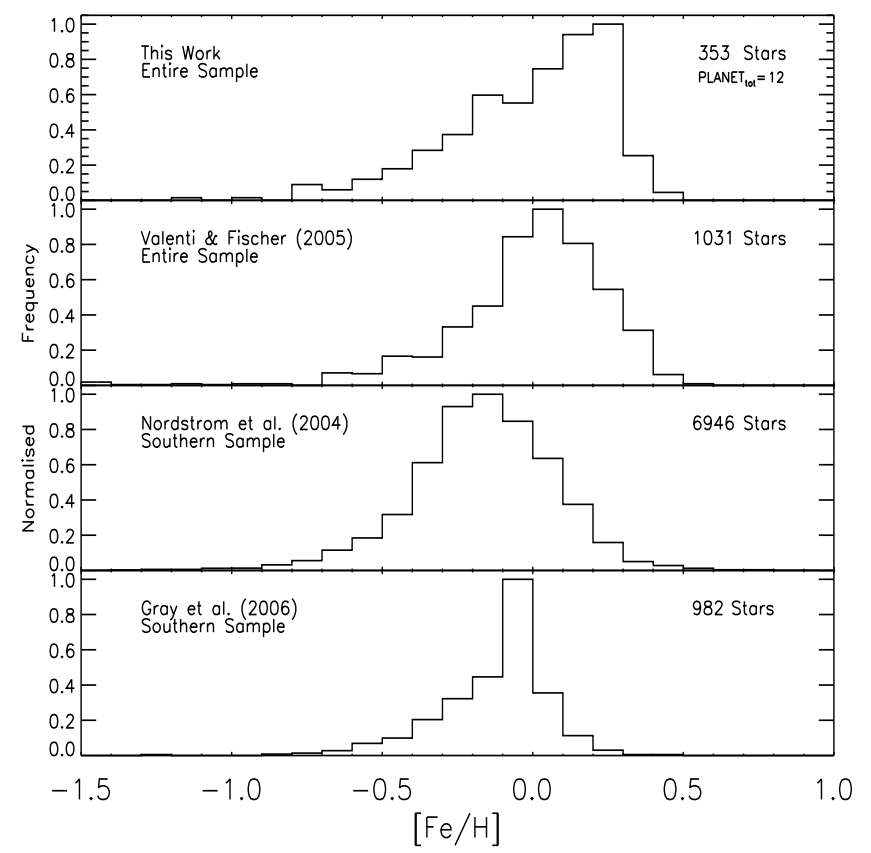

Fig. 12. Four plots showing the distributions of metallicities taken from this work (upper), Valenti \& Fischer (2005) (upper middle), Nordström et al. (2004) (lower middle) and Gray et al. (2006) (lower). Since all four distributions have significantly different sample sizes all were normalised to the peak of the distribution, with the sample sizes indicated on the plots. The labels also represent the constituent samples as the upper, lower middle and lower panels represent the southern hemisphere, whereas the upper middle panel is mostly the northern hemisphere but was included as this dataset represents the comparison sample with lowest internal errors. The upper and upper middle distributions peak around $0-0.1$ dex due to the metal-rich bias inherent in the sample selection, whereas both the middle and bottom panels should provide a better representation of the overall metallicity distribution in the southern hemisphere.

is the total number of expected planet hosting stars, following the results in Fischer \& Valenti (2005). As mentioned above, Fischer $\&$ Valenti have found that the probability of hosting a planet follows a power law increasing to $25 \%$ for stars with metallicities over +0.3 dex. By assuming this power law for the sample we have estimated the total number of planets expected to be found in this metal-rich sample to be $\sim 12$ and is shown in the upper right of the figure. Note this is for all objects not currently on any large planet search programs. Of the 137 currently discovered planets around metal-rich stars, 15 have been found to transit their host star, giving rise to a fraction of $\sim 11 \%$ that any detected planet around a metal-rich star will transit. Therefore, we expect there to be at least one transiting planet in our metal-rich sample that can be followed-up with high-resolution spectroscopy to probe the atmospheres of exoplanets. It must be noted that this only estimates the number of hot Jupiter-type planets down to a mass of $0.5 M_{\mathrm{J}}$ and a radial-velocity precision of $40 \mathrm{~ms}^{-1}$. Any additional hot Saturns or Neptunes will not be covered by this estimate and therefore the true number of detectable planets should be larger.

From the upper part of Fig. 12 it is shown that the distribution of stars in this biased sample mostly followed a Gaussian distribution with an extended metal-poor tail. The other panels in Fig. 12 show three metallicity distributions from the surveys we have compared to in Sect. 4.2. The upper middle panel shows the distribution of stars taken from Valenti \& Fischer (2005). This sample contains stars mostly from the northern hemisphere,

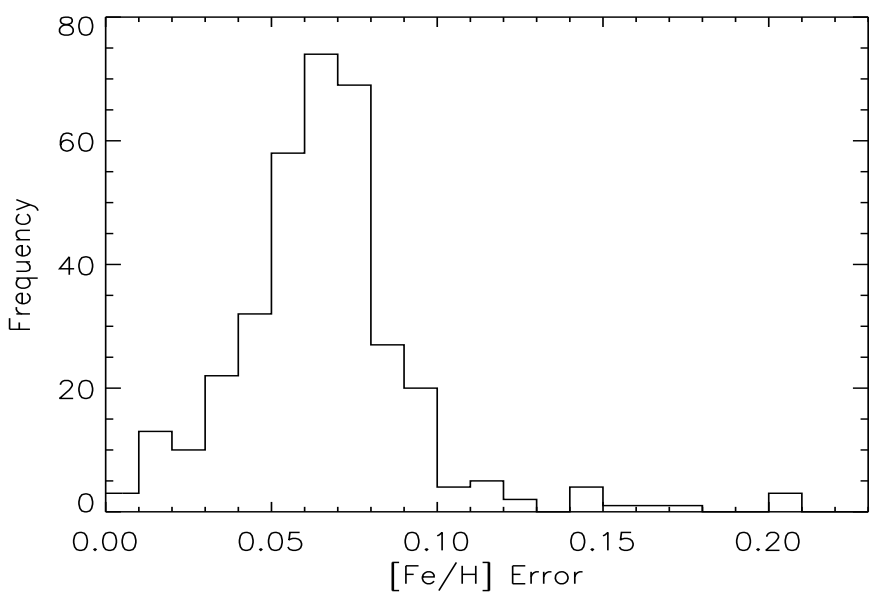

Fig. 13. The distribution of $1-\sigma$ uncertainties from the chi-squared fitting of all stars in this project. The distribution peaks at $\pm 0.065 \mathrm{dex}$, with the majority of values spread between $0.03-0.10$. There are also a number of stars with larger uncertainties, which were mostly the stars that were observed through cloud cover and have lower S/N.

however as the northern and southern distributions are similar (e.g. Gray et al. 2003; Nordström et al. 2004; Gray et al. 2006) and the methodology used was similar to the methodology used here, it can provide a useful comparison sample. The peak of this data agrees well with this work (upper panel) as it lies between $0-0.1$ dex, slightly metal-rich. This is largely again due to a bias in their sample selection where they selected all stars on current large scale planet search surveys, which itself introduces a number of metal-rich stars as most surveys now have metal-rich programs. Also the extended metal-poor tail is apparent in this data. However, when compared with the lower middle (Nordström et al. 2004) and lower (Gray et al. 2006) panels the distributions differ in where the peak lies. Both these samples are taken solely from the southern hemisphere $\left(\operatorname{dec} \leq 0^{\circ}\right)$ and both have peaks below solar metallicity, with the largest sample from Nordstöm et al. peaking between -0.1 and -0.2 . These samples are both unbiased samples as their selection criteria is volume limited out to $40 \mathrm{pc}$. Nordström et al. selected a magnitude limited sample of stars with Strömgren photometry that is complete for all $\mathrm{F}$ and $\mathrm{G}$ stars out to $40 \mathrm{pc}$ and is kinematically unbiased, whereas the Gray et al. sample is as yet incomplete but also focused on all stars earlier than M0 in the Hipparcos catalogue. Both these studies should better represent the overall distribution of stars in the galactic disk. It is clear that the Nordström et al. distribution follows a Gaussian profile with only a very small indication of a metal-poor tail, yet the Gray et al. profile shows a clear extended tail. The extended tail in the distributions arise mostly from stars from both the thick disk and the galactic halo and possibly from active K stars (Gray et al. 2006).

Figure 13 shows the distribution of all uncertainties for the metallicity fits to these stars in this sample. The uncertainties appear to follow a normal distribution peaking at $\pm 0.065 \mathrm{dex}$, which agrees well with the scatter found in the metallicities when compared to other works in the literature. However, an extended tail is found at larger uncertainty values. These are mostly comprised of low $\mathrm{S} / \mathrm{N}$ stars that were observed through cloud cover. The low $\mathrm{S} / \mathrm{N}$ means there are less photons making up each Fe line and the line profiles are not well behaved and harder to model. 


\subsection{Evolutionary phase}

To determine if any of the stars in this project are in a Maunder minimum phase of their evolution we have tried to ascertain their current evolutionary status. Baliunas \& Jastrow (1990) determined that $30 \%$ of the MW stars were in Maunder minimum, this was based purely on their low activity status. This estimate was revised to a lower percentage of $10-15 \%$ by Saar \& Baliunas (1992), which has since been complimented by other studies (Henry et al. 1996; Saar et al. 1998; Gray et al. 2003). However, Wright (2004b) has shown that these stars are all either evolved, so the application of the activity index does not apply, or are metal-poor, which alters the CaHK line wings of such stars so their indices are unreliable. Note that activity indices are calibrated for stars thought to be on the main sequence and hence the further from the main sequence a star evolves the more unreliable the index becomes. Indeed it may be necessary to re-determine the activity indices for more evolved stars. What is clear is that the Sun went through a similar activity minimum in the 17th Century and it has been estimated that the solar $S_{\mathrm{MW}}$ index decreased to a mean value of around 0.145 (Baliunas \& Jastrow 1990, currently the mean is $S_{\odot}=0.165$ ). By applying this determination to the analysis of brightness changes due to activity, Zhang et al. (1994) have determined that the Sun was $0.2-0.6 \%$ dimmer during the Maunder minimum. Stars in this study may be in a similar evolutionary phase. Figure 14 shows the distribution of stars in this survey on a colour-magnitude diagram. The $B-V$ colours and absolute magnitudes were extracted from the Hipparcos catalogue (Perryman et al. 1997). The main sequence is clear in this plot, however to obtain the true main sequence we used the model of Wright (2004b). This model was determined using a 9th-order polynomial fit to the Hipparcos main sequence, described by

$M_{V, M S}(B-V)=\sum a_{i}(B-V)^{i}$

Here, $M_{V, M S}(B-V)$ is the absolute magnitude of the main sequence as a function of colour, $a_{i}$ are the coefficients describing the polynomial, which are the following: 0.909, $6.258,-23.022,125.5537,-321.1996,485.5234,-452.3198$, $249.6461,-73.57645$ and 8.8240 . As expected the majority of stars are clustered around the main sequence fit, with a few outliers above and below. Most of the stars far below the fit are extremely metal-poor objects showing that metallicity can significantly alter a stars position on the HR-diagram. Conversely, high metal abundance will place stars above the main sequence and contribute to the spread of values around the fitted main sequence. The dashed lines represent the spread across the main sequence for a metallicity of one-third the solar metallicity (lower) and three times solar (upper) in a similar fashion to the analysis of Wright. Stars that are significantly higher than the main sequence are evolving off the main sequence and moving across to the red giant branch. Wright has shown that the majority of stars announced as being in a Maunder minima are actually evolved stars and hence the indices are not applicable to this conclusion. There are 79 stars in this study that are extremely inactive $\left(\log R_{H K}^{\prime} \leq-5.10\right)$. Other authors have claimed that stars with such properties are in a Maunder minimum phase (e.g. Henry et al. 1996; Saar et al. 1998; Gray et al. 2003). These stars are highlighted in Fig. 14 by filled circles. It is clear that the majority are located high above the main sequence and are evolving onto the giant branch, with two below and the rest close to the main sequence. To determine how many of these stars are
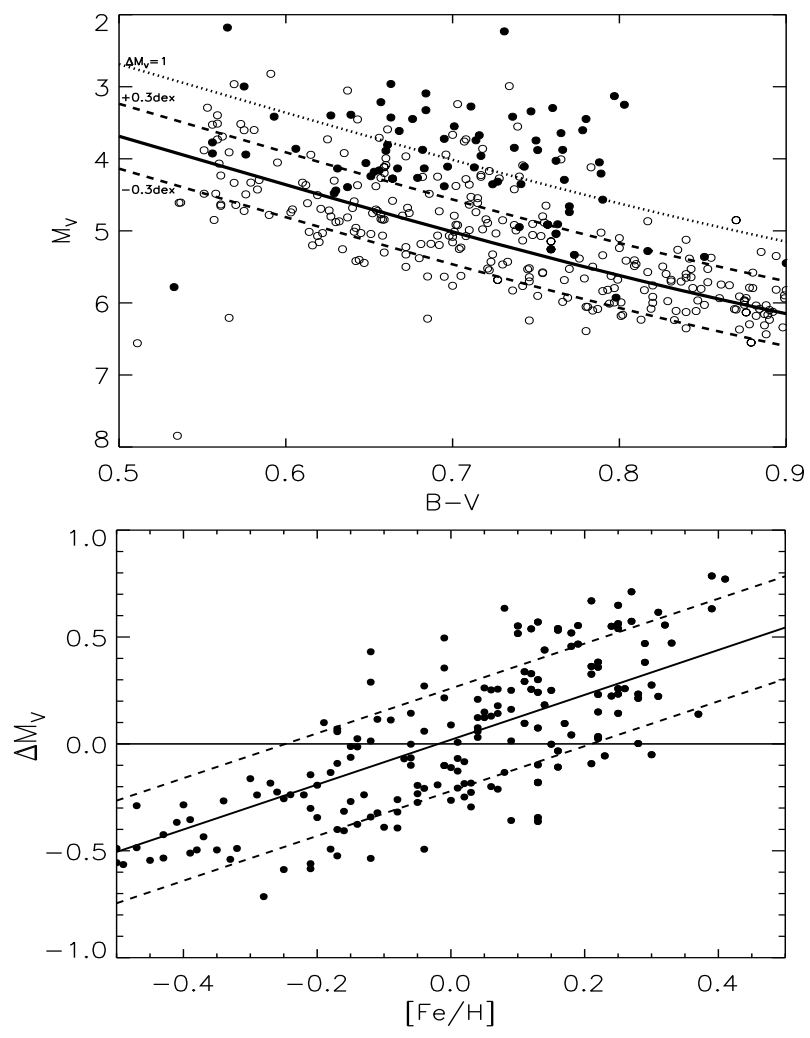

Fig. 14. The top plot shows a Hipparcos colour-magnitude diagram of all stars in this survey except for the calibration stars above a $B-V$ of 0.9. The open circles represent all the target stars in this colour space. The solid line is the empirical main sequence of Hipparcos stars determined in Wright (2004b), with the dashed lines representing the spread in the main sequence due to metallicity $( \pm 0.3 \mathrm{dex})$. The dotted line represents a difference of 1 magnitude above the main sequence and this isolates evolved stars. It is a clear a number of these stars are evolving off the main sequence. There are also a number of a stars far below the main sequence that comprise thick-disk and halo stars. The filled circles represent possible Maunder Minimum stars in this study (upper panel). The lower plot shows the distance from the fitted main sequence against each stars metallicity within a subset of the original sample. This represents all stars where the surface gravity was greater than $4.0(\log (g))$. The solid line represents the best-fit straight line with the dashed lines representing the $1 \sigma$ uncertainty boundaries. This fit can be used to correct each stars position on the main sequence for the effects of metallicity.

clearly on the main sequence we use the definition of the height above the main sequence in Wright (2004b), which is given as

$\left(\Delta M_{V}\right)_{\mathrm{MS}, B-V}=M_{V, \mathrm{MS}}(B-V)-M_{V}$.

$\left(\Delta M_{V}\right)_{\mathrm{MS}, B-V}$ is the difference between a stars absolute magnitude and the absolute magnitude of the main sequence fit (for that $B-V$ ). Equation (4) is used to determine the height above the main sequence, giving an indication if a star is evolving off the main sequence or not. Wright estimate that stars with a metallicity of +0.3 dex will lie $\sim 0.45$ mag above the main sequence, whereas stars with a metallicity of -0.3 dex will lie $\sim 0.45$ mag below the main sequence. These regions are shown as dashed lines in Fig. 14 and the majority of stars in this study are located between these two bounds, both inactive and active stars. Also shown on the plot is the region that marks $\left(\Delta M_{V}\right)_{\mathrm{MS}, B-V}=$ 1 (dotted line) which we use as a boundary for evolutionary status. Stars above this line we expect to be in an evolved state of their evolution regardless of their metallicity. Since we have 
measured the metallicities, a correction can be made to remove the metallicity component in each star's main sequence position.

The lower plot in Fig. 14 shows the distribution of stellar metallicities against distance from the main sequence. A clear trend is seen in the plot with the metal-poor objects generally found below the main sequence and metal-rich objects located above. This sequence was cleaned by focusing on stars that show no significant evidence for evolution into giant status. As mentioned above, all stars with a $\left(\Delta M_{V}\right)_{\mathrm{MS}, B-V}>1$ must be evolving off the main sequence and are removed from this sample. To ensure this is the case and to detect any evolved objects located $\left(\Delta M_{V}\right)_{\mathrm{MS}, B-V}<1$, an estimate of the surface gravity was made following the technique described in Sect. 3.3.1. Using surface gravities we were able to remove all stars with a $\log (g)$ of 3.5 or 4.0 , which with grid steps of 0.5 , should effectively remove all stars below 4.25. This is a conservative estimate but should select against any stars evolving off the main sequence and leave behind only true main sequence stars. A first fit was then made to this subset of stars to estimate where the main sequence lies and the $1 \sigma$ scatter was measured and used to perform a secondary cut, which removed all objects outside the first scatter region. This cut was used to better constrain the position of the main sequence by focusing on the region that is most populated. The thick solid line Fig. 14 (lower) shows the best-fit linear trend to this main sequence across all metallicities, after all cuts have been performed, accompanied by the $1 \sigma$ uncertainty regions (dashed lines). The trend is evident and it should be noted that the fit intersects the point of zero distance from the main sequence very close to the solar metallicity value of zero without any necessary fixing or adjustment. The relationship is described by

$$
\left(\Delta M_{V}\right)_{[\mathrm{Fe} / \mathrm{H}]}=1.050([\mathrm{Fe} / \mathrm{H}])+0.019 \text {. }
$$

The uncertainties on the fitted coefficients in Eq. (5) are 0.018 and 0.071 respectively, and the $1 \sigma$ uncertainty shown by the dashed lines in the figure is \pm 0.240 . Since the majority of stars reside within the scatter region we are confident this region is a good proxy for the main sequence in this sample. Simply by subtracting off $\left(\Delta M_{V}\right)_{[\mathrm{Fe} / \mathrm{H}]}$ from Eq. (5) from the initially determined $\left(\Delta M_{V}\right)_{\mathrm{MS}, B-V}$ in Eq. (4) we can remove the effects of metallicity on the spread in distance from the main sequence. This repositions all stars onto the main sequence irrespective of their metallicities and since all evolved objects have been removed a clean sample of possible Maunder minimum candidates can be made, based on their inactivity and distance from the main sequence. From the stars that are left we have selected those that are within the $1 \sigma$ boundaries of this fitted main sequence. From this selection six stars remain and should represent both main sequence stars and very inactive stars, all of these are listed in Table 2. Note that the distance from the main sequence $\left(\Delta M_{V}\right)$ quoted in this table is after the metallicity correction has been applied. A check of any affects due to line strength changes between metal-poor and metal-rich objects was made to test if any lines within the bandpass regions were biasing the metal-rich stars towards lower activities. One absorption line was found inside the $H$ bandpass region and none inside the $K$ bandpass region. We interpolated this line out and recomputed the activity index and found that there were no real changes in the final $\log R_{H K}^{\prime}$ activities. This result was similar for the continuum bandpass regions and therefore any line strength alterations are minimal compared with the reduction uncertainties. However, the metallicity does affect the intrinsic colour of stars and should affect the derived $\log R_{H K}^{\prime}$ value as this is colour dependent. It is also important to note that since only one
Table 2. Six possible Maunder minimum stars.

\begin{tabular}{cccccc}
\hline \hline HD & $B-V$ & $V$ & $\Delta M_{V}$ & $\log R_{H K, \mathrm{FEROS}}^{\prime}$ & {$[\mathrm{Fe} / \mathrm{H}]$} \\
\hline HD 8446 & 0.661 & 8.16 & -0.15 & -5.17 & -0.35 \\
HD 9175 & 0.656 & 8.01 & -0.24 & -5.12 & -0.12 \\
HD 22104 & 0.679 & 8.32 & 0.07 & -5.15 & 0.15 \\
HD 31827 & 0.770 & 8.26 & 0.07 & -5.11 & -0.34 \\
HD 84501 & 0.663 & 8.26 & -0.03 & -5.12 & -0.22 \\
HD 90722 & 0.724 & 7.88 & -0.15 & -5.10 & -0.20 \\
\hline
\end{tabular}

measurement has been made for these objects in this study, it is unsure whether or not they will remain stable over a number of years or will undergo any cyclical variation. If we assume a Gaussian distribution to describe the cyclical activity variations and take the stars with the potential to scatter above the -5.10 activity boundary (i.e. stars within the range -5.25 to -5.10 which represents a maximum cycle of 0.15 in $\log R_{H K}^{\prime}$ and is consistent with the Sun's cycle) then four of the six stars are expected to be normal stars undergoing their usual activity cycle at the $1 \sigma$ level of confidence. Of the entire sample of 353 stars, this leads to a fraction of $\sim 2 \pm 1 \%$ and hence the Sun should spend only a few percent of its main sequence lifetime in a Maunder minimum like state. It should be noted that the sample is biased towards the most metal-rich objects in the solar neighbourhood. This selection will skew the final estimate of Maunder minimum stars as it should be biased towards younger more active objects.

\subsection{Benchmark target list}

The main aim of this study was to determine accurate chromospheric activity and metallicity values for a number of close-by, bright stars in the southern hemisphere. The metal-rich subset of these will provide excellent candidates for planet search studies searching for hot giant planets. Figure 12 (upper) shows the distribution of metallicities in this sample. About $30 \%$ of stars have metallicities greater than 0.1 dex, of which $15 \%$ have metallicities greater than 0.2 dex and it is these that represent the best targets for radial-velocity follow-up. First epoch radial-velocities have already been acquired for a number of these stars in ESO Period 76 with the HARPS instrument.

The chromospheric activities also play an important role in the nature of the objects that can be detected. Figure 15 shows the distribution of activities across all metallicity space. Only a few of the metal-rich candidates are active and will be removed from the final candidate list, whereas most of the stars above +0.1 dex in $[\mathrm{Fe} / \mathrm{H}]$ have $\log R_{H K}^{\prime}$ values less than -4.5 . Since the level of radial-velocity noise (aka. jitter) is correlated with activity (Saar et al. 1998; Santos et al. 2000; Wright 2005) these inactive stars allow higher precision radial-velocities to be efficiently measured. The potential to access lower amplitude signals allows access to objects of lower masses and as the mass function rises towards lower masses following a power law of -1.8 (Grether \& Lineweaver 2006), the probability of planet detection significantly increases. The final target list will constitute a subset of stars taken from Table 4, which shows the Johnson $V$ magnitudes and $B-V$ colours from Hipparcos, along with the derived $S$ and $\log R_{H K}^{\prime}$ indices and final $[\mathrm{Fe} / \mathrm{H}]$ abundances. The subset will follow the criteria explained above.

\section{Conclusions}

Using the high-resolution echelle data from the FEROS instrument we extracted chromospheric activities and metallicities for 
Table 3. Derived activity and metallicity values of all stars used to calibrate onto the Mt. Wilson system of measurements.

\begin{tabular}{ccccccccc}
\hline \hline HD & $B-V$ & $V$ & $S_{\text {FEROS }, \mathrm{a}}$ & $S_{\text {FEROS }, \mathrm{b}}$ & $S_{\mathrm{MW}}$ & $\log R_{H K, \mathrm{FEROS}}^{\prime}$ & $\log R_{H K, \mathrm{MW}}^{\prime}$ & {$[\mathrm{Fe} / \mathrm{H}]$} \\
\hline Calibrators & & & & & & & & \\
HD 1835 & 0.659 & 6.39 & 0.291 & $0.350 \pm 0.003$ & 0.347 & -4.43 & -4.44 & $0.21 \pm 0.03$ \\
HD 3443AB & 0.715 & 5.57 & 0.142 & $0.180 \pm 0.001$ & 0.186 & -4.92 & -4.89 & $-0.01 \pm 0.05$ \\
HD 3795 & 0.718 & 6.14 & 0.119 & $0.153 \pm 0.001$ & 0.156 & -5.06 & -5.04 & $-0.65 \pm 0.05$ \\
HD 9562 & 0.639 & 5.75 & 0.106 & $0.138 \pm 0.001$ & 0.144 & -5.16 & -5.11 & $0.19 \pm 0.14$ \\
HD 10700 & 0.727 & 3.49 & 0.140 & $0.178 \pm 0.001$ & 0.173 & -4.93 & -4.95 & $-0.38 \pm 0.04$ \\
HD 10700 & 0.727 & 3.49 & 0.142 & $0.180 \pm 0.001$ & 0.173 & -4.92 & -4.95 & $-0.35 \pm 0.04$ \\
HD 22484 & 0.575 & 4.29 & 0.118 & $0.153 \pm 0.001$ & 0.147 & -5.02 & -5.06 & $-0.14 \pm 0.02$ \\
HD 30495 & 0.632 & 5.49 & 0.234 & $0.286 \pm 0.002$ & 0.292 & -4.54 & -4.54 & $0.03 \pm 0.03$ \\
\hline
\end{tabular}

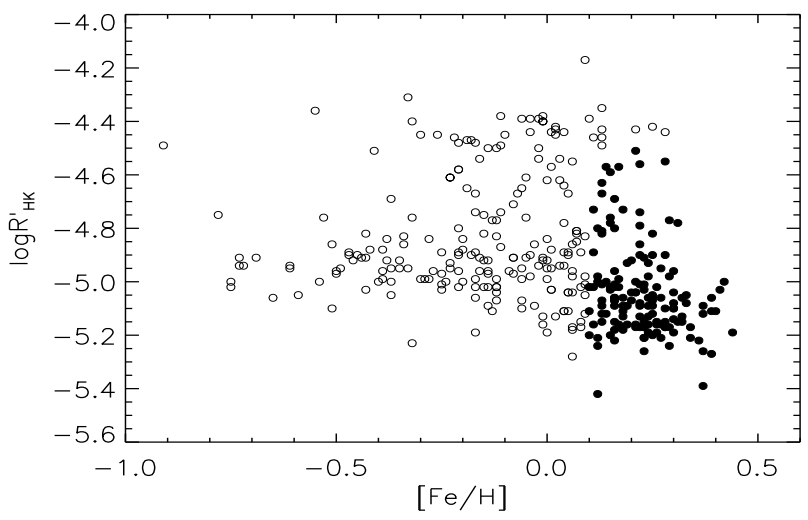

Fig. 15. Iron abundance as a function of chromospheric activity of the stars in this sample. The filled circles represent all stars that will compose our metal-rich target list. While most stars above +0.1 dex are inactive, a few have activities above -4.5 .

353 bright stars in the Southern hemisphere. We have shown the activity distribution can be described well by a bimodal function which agrees with previous work in both the northern and southern hemispheres. The sample is dominated by $\mathrm{G}$ stars $\left(T_{\mathrm{EFF}} \sim\right.$ $6000 \mathrm{~K})$ and the activity distribution peaks around -5.0 . The $\mathrm{K}$ stars have an active to inactive peak ratio close to 1 , compared with the $\mathrm{G}$ stars peak-to-peak ratio of 0.2. The metallicity distribution peaks around 0.1 dex, contrary to unbiased surveys which peak around -0.1 dex. Over 90 stars conform to our radial-velocity target selection criteria and will be observed to look for signatures indicative of planets. The uncertainties follow a Gaussian distribution peaking at \pm 0.085 dex with a high uncertainty tail of objects due to stars with low S/N spectra. The values are in agreement with similar works in the literature, such as Valenti \& Fischer (2005), with an rms scatter of $\sim 0.05$ dex.

Acknowledgements. We would like to thank Pierre Maxted for his suggestions regarding various aspects of the paper, along with the anonymous referee for their various suggestions. We would also like to acknowledge the help from Patrick Francois whilst observing with the FEROS instrument. We also acknowledge the use of the Vizier and Simbad databases.

\section{References}

Alonso, A., Arribas, S., \& Martinez-Roger, C. 1996, A\&A, 313, 873

Asplund, M., Nordlund, Å., Trampedach, R., Allende Prieto, C., \& Stein, R. F. 2000, A\&A, 359, 729

Baliunas, S., \& Jastrow, R. 1990, Nature, 348, 520

Baliunas, S. L., Donahue, R. A., Soon, W., Gilliland, R., \& Soderblom, D. R. 1995, BAAS, 27, 839

Bigot, L., \& Thévenin, F. 2006, MNRAS, 372, 609

Blackwell, D. E., \& Lynas-Gray, A. E. 1994, A\&A, 282, 899

Bond, J. C., Tinney, C. G., Butler, R. P., et al. 2006, MNRAS, 370, 163

Carpenter, J. M. 2001, AJ, 121, 2851
Duncan, D. K., Vaughan, A. H., Wilson, O. C., et al. 1991, ApJS, 76, 383 Edvardsson, B., Andersen, J., Gustafsson, B., et al. 1993, A\&A, 275, 101 Fischer, D. A., \& Valenti, J. 2005, ApJ, 622, 1102

Gonzalez, G. 1997, MNRAS, 285, 403

Gray, D. F. 2005, The Observation and Analysis of Stellar Photospheres, 3rd Ed., ed. D. F. Gray (Cambridge, UK: Cambridge University Press)

Gray, R. O., Corbally, C. J., Garrison, R. F., McFadden, M. T., \& Robinson, P. E. 2003, AJ, 126, 2048

Gray, R. O., Corbally, C. J., Garrison, R. F., et al. 2006, AJ, 132, 161

Grether, D., \& Lineweaver, C. H. 2006, ApJ, 640, 1051

Gurtovenko, E. A., \& Kostik, R. I. 1998, Main Astronomical Observatory NAS Ukraine, 3E

Hall, J. C., Lockwood, G. W., \& Skiff, B. A. 2007, AJ, 133, 862

Hauck, B., \& Mermilliod, M. 1998, A\&AS, 129, 431

Haywood, M. 2002, MNRAS, 337, 151

Henry, G. W., Donahue, R. A., \& Baliunas, S. L. 2002, ApJ, 577, L111

Henry, T. J., Soderblom, D. R., Donahue, R. A., \& Baliunas, S. L. 1996, AJ, 111, 439

Jenkins, J. S., Jones, H. R. A., Tinney, C. G., et al. 2006, MNRAS, 372, 163

Jones, H. R. A., Butler, R. P., Marcy, G. W., \& Tinney, C. G. 2002, MNRAS, 337,1170

Kaufer, A., Stahl, O., Tubbesing, S., et al. 1999, The Messenger, 95, 8

Kurucz, R. 1993, ATLAS9 Stellar Atmosphere Programs and $2 \mathrm{~km} \mathrm{~s}^{-1}$ grid. Kurucz CD-ROM, 13 (Cambridge, Mass.: Smithsonian Astrophysical Observatory)

Kurucz, R. L. 1979, ApJS, 40, 1

Kurucz, R. L. 1992, Rev. Mex. Astron. Astrofis., 23, 45

Lockwood, G. W., Skiff, B. A., Henry, G. W., et al. 2007, ApJS, 171, 260

Middelkoop, F. 1982a, A\&A, 107, 31

Middelkoop, F. 1982b, A\&A, 113, 1

Moore, C. E. 1956, Vistas in Astron., 2, 1209

Nordström, B., Mayor, M., Andersen, J., et al. 2004, A\&A, 418, 989

Noyes, R. W., Hartmann, L. W., Baliunas, S. L., Duncan, D. K., \& Vaughan, A. H. 1984a, ApJ, 279, 763

Olsen, E. H. 1984, A\&AS, 57, 443

Pavlenko, Y. V. 1991, Sov. Astron., 35, 212

Pavlenko, Y. V. 2000, Astron. Rep., 44, 219

Pavlenko, Y. V., Rebolo, R., Martin, E. L., \& Garcia Lopez, R. J. 1995, A\&A, 303, 807

Perryman, M. A. C., Lindegren, L., Kovalevsky, J., et al. 1997, A\&A, 323, L49 Queloz, D., Henry, G. W., Sivan, J. P., \& Baliunas, S. L. 2001, A\&A, 379, 279 Rieutord, M., Roudier, T., Ludwig, H.-G., Nordlund, Å., \& Stein, R. 2001, A\&A, 377, L14

Rutten, R. G. M. 1984, A\&A, 130, 353

Saar, S. H., \& Baliunas, S. L. 1992, in The Solar Cycle, ed. K. L. Harvey, ASP Conf. Ser., 27, 150

Saar, S. H., Butler, R. P., \& Marcy, G. W. 1998, ApJ, 498, L153

Santos, N. C., Mayor, M., Naef, D., et al. 2000, A\&A, 361, 265

Santos, N. C., Israelian, G., Mayor, M., Rebolo, R., \& Udry, S. 2003, VizieR Online Data Catalog, 339, 80363

Santos, N. C., Israelian, G., \& Mayor, M. 2004, A\&A, 415, 1153

Schuster, W. J., \& Nissen, P. E. 1989, A\&A, 221, 65

Smalley, B., \& Kupka, F. 1997, A\&A, 328, 349

Smith, R. C. 1995, Observational Astrophysics, ed. R. C. Smith (Cambridge, UK: Cambridge University Press), 467

Tinney, C. G., McCarthy, C., Jones, H. R. A., et al. 2002, MNRAS, 332, 759

Twarog, B. A., Anthony-Twarog, B. J., \& Tanner, D. 2002, AJ, 123, 2715

Valenti, J. A., \& Fischer, D. A. 2005, ApJS, 159, 141

Wright, J. T. 2004a, AJ, 128, 1273

Wright, J. T. 2004b, AJ, 128, 1273

Wright, J. T. 2005, PASP, 117, 657

Zhang, Q., Soon, W. H., Baliunas, S. L., et al. 1994, ApJ, 427, L111 